\title{
ON NONSEPARABLE BANACH SPACES
}

\author{
BY
}

SPIROS A. ARGYROS

\begin{abstract}
Combining combinatorial methods from set theory with the functional structure of certain Banach spaces we get some results on the isomorphic structure of nonseparable Banach spaces. The conclusions of the paper, in conjunction with already known results, give complete answers to problems of the theory of Banach spaces. An interesting point here is that some questions of Banach spaces theory are independent of Z.F.C. So, for example, the answer to a conjecture of Pelczynski that states that the isomorphic embeddability of $L^{1}\{-1,1\}^{\alpha}$ into $X^{*}$ implies, for any infinite cardinal $\alpha$, the isomorphic embedding of $l_{\alpha}^{1}$ into $X$, gets the following form:

if $\alpha=\omega$, has been proved from Pelczynski;

if $\alpha>\omega^{+}$, the proof is given in this paper;

if $\alpha=\omega^{+}$, in Z.F.C. + C.H., an example discovered by Haydon gives a negative answer;

if $\alpha=\omega^{+}$, in Z.F.C. +$\rceil$C.H. + M.A., is also proved in this paper.
\end{abstract}

Introduction. In this paper we study certain problems on the isomorphic structure of Banach spaces, some of which are conjectures or problems posed by researchers in the field. Our methods are to a large extent infinitary combinatorics; an unexpected by-product of our results, together with Haydon's counterexample, is that the answer to most of these problems and conjectures is independent of Z.F.C. for $\alpha=\omega^{+}$, while they have an affirmative answer for higher cardinals. More precisely our results can be summarised as follows.

TheOREM A (3.1). If $\alpha>\omega^{+}, X$ is a Banach space such that $L^{1}\{-1,1\}^{\alpha}$ is isomorphic to a subspace of $X^{*}$, then $l_{\alpha}^{1}$ is isomorphic to a subspace of $X$.

THEOREM B (6.11). Assume Martin's axiom and the negation of the continuum hypothesis. If $X$ is a Banach space such that $L^{1}\{-1,1\}^{\omega^{+}}$is isomorphic to a subspace of $X^{*}$, then $X$ contains an isomorphic copy of $l_{\Omega^{+}}^{1}$.

Theorems A and B give an affirmative answer to a conjecture of Pełczynski [13]; in conjunction with a counterexample by Haydon, it follows that Pekcynski's conjecture is independent of Z.F.C. for $\alpha=\omega^{+}$. The proofs of Theorems A and B are given in $\$ \S 3$ and 6, below, respectively.

THEOREM C (4.2). If $\alpha>\omega^{+}, X$ is a Banach space, $\lambda$ is an arbitrary (not necessarily finite) measure, and $T: L^{1}\{-1,1\}^{\alpha} \rightarrow X^{*}, U: X^{*} \rightarrow L^{1}(\lambda)$ are bounded linear operators such that $U \circ T$ is an isomorphic embedding, then $Z_{\alpha}$ is isomorphic to

Received by the editors December 12, 1979 and, in revised form, March 16, 1981.

1980 Mathematics Subject Classification. Primary 46B99. 
a subspace of $X$, where

$$
Z_{\alpha}=\left(\sum_{\xi<\alpha} \oplus Z_{\xi}\right)_{1}, \quad Z_{\xi}=\left(\sum_{n=1}^{\infty} \oplus l^{\infty}(n)\right)_{1} \text { for } \xi<\alpha .
$$

TheOREM D (6.3). Assume Martin's axiom and the negation of the continuum hypothesis. If $X$ is a Banach space, $\lambda$ is an arbitrary (not necessarily finite) measure, and $T: L^{1}\{-1,1\}^{\omega^{+}} \rightarrow X^{*}, U: X^{*} \rightarrow L^{1}(\lambda)$ are bounded linear operators such that $U \circ T$ is an isomorphic embedding, then $Z_{\omega^{+}}$is isomorphic to a subspace of $X$.

Theorems C and D imply an affirmative answer to a problem by Hagler and Stegall [7]; in conjunction with the counterexample by Haydon, mentioned above, it follows that the Hagler-Stegall conjecture is independent of Z.F.C. for $\alpha=\omega^{+}$. The proofs of Theorems $\mathrm{C}$ and $\mathrm{D}$ are given in $\$ \S 4$ and 6 , below, respectively.

TheOrem E (5.2). Let $\alpha$ be a strongly $\omega^{+}$inaccessible with $\operatorname{cf}(\alpha)>\omega^{+}$; if $X$ is a subspace of $L^{\infty}(\mu)$ for some finite measure $\mu$ with $\operatorname{dim} X=\alpha$ then $l_{\alpha}^{1}$ is isomorphic to a subspace of $X$.

Theorem E improves some previous results about the universal embedding of $l_{\alpha}^{1}$ into the subspaces of $L^{\infty}(\mu)$ spaces. The proof of this theorem is given in $\S 5$ below.

THEOREM F (7.3). Let $X$ be an injective Banach space of dimension $\alpha$.

The following are equivalent:

(a) $l_{\alpha}^{1}$ is isomorphic to a subspace of $X$;

(b) $X^{*}$ is isomorphic to $\left(C\{-1,1\}^{\alpha}\right)^{*}$.

Statements (a) and (b) are two independent problems posed by Rosenthal [14]. The proof of Theorem $F$ is given in $\S 7$. Also in the same section we prove the following.

TheOrem G (7.1). Assume Martin's axiom and the negation of the continuum hypothesis. If $X$ is a Banach space, $\lambda$ is an arbitrary (not necessarily finite) measure, and $L^{1}(\lambda)$ is isomorphic to $X^{*}$, then there is a set $\Delta$ and a set of cardinals $\left\{\alpha_{\gamma}: \gamma \in \Gamma\right\}$ such that $L^{1}(\lambda)$ is isomorphic to

$$
l^{1}(\Delta) \oplus\left(\sum_{\gamma \in \Gamma} \oplus C\left(\{-1,1\}^{\alpha_{\gamma}}\right)^{*}\right)_{1}
$$

Theorem $G$ gives an affirmative answer to a conjecture of Rosenthal [14]; in conjunction with the counterexample by Haydon, mentioned above, it follows that Rosenthal's conjecture is independent of Z.F.C.

Acknowledgement. The author thanks Professor S. Negrepontis for useful conversations on the subject of this paper. He is also grateful to Dr. R. Haydon for his valuable help during the preparation of the paper. In particular his suggestions allowed the author to simplify the proofs of some results of the paper.

0. Preliminaries. The ordinals are defined in such a way that an ordinal is the set of smaller ordinals. A cardinal is an ordinal not in one-to-one correspondence with any smaller ordinal. The cofinality of a cardinal $\alpha$, denoted $\operatorname{cf}(\alpha)$, is the least 
cardinal $\beta$ such that $\alpha$ is the cardinal sum of $\beta$ many cardinals, each smaller than $\alpha$. A cardinal $\alpha$ is regular if $\alpha=\operatorname{cf}(\alpha)$, and singular if $\operatorname{cf}(\alpha)<\alpha$. The least cardinal strictly greater than $\beta$ is denoted by $\beta^{+}$. A cardinal $\alpha$ is a successor cardinal if it is of the form $\alpha=\beta^{+}$for some $\beta$; every successor cardinal is regular. The cardinality of the natural numbers is denoted by $\omega$. The cardinality of a set $A$ is denoted by $|A|$.

We denote by $\mathscr{P}(\alpha)$ (resp., $\left.\mathscr{P}_{\kappa}(\alpha)\right)$ the set of subsets of $\alpha$ (resp., the set of subsets of $\alpha$ of cardinality less than $\kappa$ ). The cardinality of $\mathscr{P}(\alpha)$ is denoted by $2^{\alpha}$.

The continuum hypothesis is the statement $\omega^{+}=2^{\omega}$. The generalized continuum hypothesis is the statement $\alpha^{+}=2^{\alpha}$ for all infinite cardinals $\alpha$.

If $\alpha$ is a cardinal, then $\alpha$ is called strongly $\omega^{+}$-inaccessible if $\beta^{\omega}<\alpha$ for any $\beta<\alpha$, and we write $\alpha \gg \omega^{+}$.

For set theoretic background, we refer the reader to [4].

By $L^{1}(\mu)$ we denote the Banach space $L^{1}(\Omega, \Sigma, \mu)$ of equivalence classes of complex $\mu$-measurable functions such that $\|f\|_{1}=\int|f| d \mu<\infty$ (where $\Omega$ is a set, $\Sigma$ is a $\sigma$-algebra of subsets of $\Omega$, and $\mu$ is a positive measure on $\Sigma$ ); by $L^{\infty}(\mu)$ we denote the Banach space $L^{\infty}(\Omega, \Sigma, \mu)$ of equivalence classes of complex $\mu$-measurable $\mu$-essentially bounded functions, with $\|f\|_{\infty}=\mu$-ess. $\sup _{\omega \in \Omega}|f(\omega)|$. If $\Omega=$ $\{-1,1\}^{I}, \Sigma$ is the $\sigma$-algebra of Borel sets of $\Omega$, and $\mu$ is the (unique) probability Haar measure on the (compact abelian) topological group $\{-1,1\}^{I}$, we denote $L^{1}(\Omega, \Sigma, \mu)\left(\right.$ resp. $\left.L^{\infty}(\Omega, \Sigma, \mu)\right)$ by $L^{1}\{-1,1\}^{I}\left(\right.$ resp. $\left.L^{\infty}\{-1,1\}^{I}\right)$; the measure $\mu$ is alternatively described as follows: Let $\nu$ be the measure on $\{-1,1\}$ defined by $\nu\{-1\}=\nu\{1\}=\frac{1}{2}$, and let $\mu$ be the product measure of the $\mu_{i}$, with $\mu_{i}=\nu$ for all $i \in I$. If $\varnothing \neq N \subset I$, we denote by $\mu_{N}$ the probability Haar measure on $\{-1,1\}^{N}$.

If $Z$ is a Banach space, $Z^{*}$ denotes its dual; if $T: Z \rightarrow X$ is a bounded linear operator, then $T^{*}: X^{*} \rightarrow Z^{*}$ denotes the conjugate operator.

We denote by $M(S)$ the space of all regular finite complex-valued Borel measures on $S$; we identify $C(S)^{*}$ with $M(S)$ by the Riesz representation theorem. Moreover, if $\mu$ is a positive element of $M(S)$, we identify $L^{1}(\mu)$ with the (complemented) subspace of $M(S)$ consisting of all measures $\lambda$ with $\lambda$ absolutely continuous with respect to $\mu$, by the Radon-Nikodym-Lebesgue theorem.

We identify $L^{\infty}(\mu)$ with $\left(L^{1}(\mu)\right)^{*}$, for any $\sigma$-finite measure space.

Given a set $\Gamma, l^{1}(\Gamma)$ denotes the Banach space of all complex-valued functions $f$ defined on $\Gamma$ such that $\Sigma_{\gamma \in \Gamma}|f(\gamma)|<\infty$, with $\|f\|=\Sigma_{\gamma \in \Gamma}|f(\gamma)|$. By the canonical basis of $l^{1}(\Gamma)$ we refer to $\left\{e_{\gamma}: \gamma \in \Gamma\right\}$, where $e_{\gamma}(\delta)=1$ if $\gamma=\delta, e_{\gamma}(\delta)=0$ if $\gamma \neq \delta$, for $\gamma \in \Gamma$.

A subset $\left\{b_{\gamma}: \gamma \in \Gamma\right\}$ of a Banach space $Z$ is said to be equivalent to the canonical basis of $l^{1}(\Gamma)$ if the correspondence $T:\left\{e_{\gamma}: \gamma \in \Gamma\right\} \rightarrow\left\{b_{\gamma}: \gamma \in \Gamma\right\}$, defined by $T e_{\gamma}=b_{\gamma}$ for $\gamma \in \Gamma$, can be extended to an isomorphism of $l^{1}(\Gamma)$ with the closed linear span of $\left\{b_{\gamma}: \gamma \in \Gamma\right\}$. If $|\Gamma|=\alpha$, we denote $l^{1}(\Gamma)$ by $l_{\alpha}^{1}$; if $|\Gamma|=\omega$, we denote $l^{1}(\Gamma)$ by $l^{1}$.

Given a family $\left\{X_{i}: i \in I\right\}$ of Banach spaces, we denote by $\left(\Sigma_{i \in I} \oplus X_{i}\right)_{1}$ the Banach space of all $x=\left(x_{i}: i \in I\right)$, with $x_{i} \in X_{i}$ for $i \in I$, such that $\|x\|=$ $\Sigma_{i \in I}\left\|x_{i}\right\|_{i}<\infty$. 


\section{Some combinatorial results.}

1.1. LEMMA ERDös-RADO. Let $\alpha>\omega$ be a regular cardinal and $\phi: \alpha \rightarrow \mathscr{P}_{\omega}(\alpha) a$ function. Then there are $A \subset \alpha$ and $N \subset \alpha$ such that

(i) $|A|=\alpha$, and

(ii) for every $\xi_{1}, \xi_{2} \in A, \xi_{1} \neq \xi_{2}$,

$$
\phi\left(\xi_{1}\right) \cap \phi\left(\xi_{2}\right)=N .
$$

1.2. LEMMA [3]. Let $\alpha$ be a singular cardinal with $\operatorname{cf}(\alpha)>\omega$ and $\phi: \alpha \rightarrow \mathscr{P}_{\omega}(\alpha) a$ function. Then there are

(a) a family $\left\{A_{\sigma}: \sigma<\operatorname{cf}(\alpha)\right\}$ of pairwise disjoint subsets of $\alpha$,

(b) a family $\left\{N_{\sigma}: \sigma<\operatorname{cf}(\alpha)\right\}$ of subsets of $\alpha$ and $N$ a subset of $\alpha$ such that

(i) $\left|\cup_{\sigma<\operatorname{cf}(\alpha)} A_{\sigma}\right|=\alpha$,

(ii) if $\sigma<\operatorname{cf}(\alpha)$ and $\xi_{1}, \xi_{2} \in A_{\sigma}$, with $\xi_{1} \neq \xi_{2}, \phi\left(\xi_{1}\right) \cap \phi\left(\xi_{2}\right)=N_{\sigma}$, and

(iii) if $\sigma_{1}<\sigma_{2}<\operatorname{cf}(\alpha)$ and $\xi_{1} \in A_{\sigma_{1}}, \xi_{2} \in A_{\sigma_{2}}$ then $\phi\left(\xi_{1}\right) \cap \phi\left(\xi_{2}\right)=N$.

1.3. THEOREM (HAJNAL) [8]. Let $\alpha>\omega^{+}$be a cardinal and $\phi: \alpha \rightarrow \mathscr{P}_{\omega^{+}}(\alpha) a$ function; then there is $A \subset \alpha$, with $|A|=\alpha$, such that if $\xi_{1}, \xi_{2} \in A$, and $\xi_{1} \neq \xi_{2}$, then $\xi_{1} \notin \phi\left(\xi_{2}\right)$.

In the rest of this section we present some combinatorial consequences of the above theorems.

1.4. Proposition. Let $\alpha>\omega^{+}$be a cardinal and $\phi: \alpha \rightarrow \mathscr{P}_{\omega^{+}}(\alpha)$ a function.

Let also $\left\{W_{n}: n<\omega\right\}$ be a partition of $\alpha$ into pairwise disjoint sets such that $\left|W_{n}\right|=\alpha$ for all $n<\omega$.

Then there is $A \subset \alpha$ such that

(i) $\left|A \cap W_{n}\right|=\alpha$ for all $n<\omega$, and

(ii) if $\xi_{1}, \xi_{2} \in A, \xi_{1} \neq \xi_{2}$, then $\xi_{1} \notin \phi\left(\xi_{2}\right)$.

Proof. For each $n<\omega$ we consider a well-ordering of $W_{n}$; let $W_{n}=\left\{\zeta_{\xi}^{n}\right.$ : $\xi<\alpha\}$, and we define a function $f: \alpha=\cup_{n<\omega} W_{n} \rightarrow \alpha$ by the rule $f\left(\zeta_{\xi}^{n}\right)=\xi$.

We set $F(\xi)=f\left(\cup\left\{\phi(\zeta): \zeta \in f^{-1}(\xi)\right\}\right)$. Using Hajnal's theorem we choose $A^{\prime} \subset \alpha$ such that $\left|A^{\prime}\right|=\alpha$ and, if $\xi_{1}, \xi_{2} \in A^{\prime}, \xi_{1} \neq \xi_{2}$, then $\xi_{1} \notin F\left(\xi_{2}\right)$. This implies that

$$
f^{-1}\left(\left\{\xi_{1}\right\}\right) \cap f^{-1}\left(F\left(\xi_{2}\right)\right)=\varnothing,
$$

and from this if $\zeta_{\xi_{1}}^{n_{1}} \in f^{-1}\left(\left\{\xi_{1}\right\}\right)$ and $\zeta_{\xi_{2}}^{n_{2}} \in f^{-1}\left(\left\{\xi_{2}\right\}\right)$, then $\zeta_{\xi_{1}}^{n_{1}} \notin \phi\left(\zeta_{\xi_{2}}^{n_{2}}\right)$. Now using transfinite induction we choose a subset $A$ of $\alpha$ such that

(i) $A \subset \cup\left\{f^{-1}(\{\xi\}): \xi \in A^{\prime}\right\}$,

(ii) $\left|A \cap W_{n}\right|=\alpha$ for all $n<\omega$,

(iii) if $\zeta_{\xi}^{n} \in A$ then

$$
A \cap\left(f^{-1}(\xi) \backslash\left\{\zeta_{\xi}^{n}\right\}\right)=\varnothing .
$$

This set $A$ is the desired one. 
1.5. Proposition. Let $\alpha>\omega^{+}$be a cardinal and $\phi: \alpha \rightarrow \mathscr{P}_{\omega^{+}}(\alpha), f: \alpha \rightarrow \mathscr{P}(\alpha)$ be functions such that if $\xi_{1}<\xi_{2}<\alpha$ then $f\left(\xi_{1}\right) \cap f\left(\xi_{2}\right)=\varnothing$. Then there is an $A \subset \alpha$, with $|A|=\alpha$, such that if $\xi_{1}, \xi_{2} \in A$, with $\xi_{1} \neq \xi_{2}$, then

$$
\phi\left(\xi_{1}\right) \cap f\left(\xi_{2}\right)=\varnothing \text {. }
$$

Proof. W.l.o.g. assume that $f(\xi) \neq \varnothing$ for all $\xi<\alpha$.

Let $F: \alpha \rightarrow \alpha$ be a function such that if $\zeta<\alpha$ and $\zeta \in f(\xi)$ for some $\xi<\alpha$, then $F(\zeta)=\xi$; otherwise $F(\zeta)=\zeta$.

We define $h: \alpha \rightarrow \mathscr{P}_{\omega^{+}}(\alpha)$ by the rule $h(\xi)=F(\phi(\xi))$; we apply Hajnal's theorem and we find a set $A$, subset of $\alpha$, with $|A|=\alpha$, such that if $\xi_{1}, \xi_{2} \in A$, and $\xi_{1} \neq \xi_{2}$, then $\xi_{1} \notin h\left(\xi_{2}\right)$.

It is easy to verify that for $\xi_{1}, \xi_{2}$, as above, $f\left(\xi_{1}\right) \cap \phi\left(\xi_{2}\right)=\varnothing$.

The next proposition is a combination of Lemmas 1.1, 1.2 and the above proposition.

1.6. Proposition. Let $\alpha \gg \omega^{+}$be a cardinal with $\operatorname{cf}(\alpha)>\omega^{+}$, and $\phi: \alpha \rightarrow \mathscr{P}_{\omega^{+}}(\alpha)$, $f: \alpha \rightarrow \mathscr{P}_{\omega}(\alpha)$ be functions.

Then there are

(a) a family $\left\{A_{\sigma}: \sigma<\operatorname{cf}(\alpha)\right\}$ of pairwise disjoint subsets of $\alpha$,

(b) a family $\left\{N_{\sigma}: \sigma<\operatorname{cf}(\alpha)\right\}$ of finite subsets of $\alpha$, and $N$ finite set such that

(i) $\left|\cup_{\sigma<\mathrm{cf}(\alpha)} A_{\sigma}\right|=\alpha$,

(ii) if $\sigma<\operatorname{cf}(\alpha)$ and $\xi_{1}, \xi_{2} \in A_{\sigma}, \xi_{1} \neq \xi_{2}$, then $\phi\left(\xi_{1}\right) \cap f\left(\xi_{2}\right) \subset N_{\sigma}$, and

(iii) if $\sigma_{1}<\sigma_{2}<\operatorname{cf}(\alpha), \xi_{1} \in A_{\sigma_{1}}, \xi_{2} \in A_{\sigma_{2}}$, then $\phi\left(\xi_{1}\right) \cap f\left(\xi_{2}\right) \subset N$.

1.7. Proposition. Let $\alpha>\omega^{+}$be a cardinal and $\phi: \alpha \rightarrow \mathscr{P}_{\omega^{+}}(\alpha), f: \alpha \rightarrow \mathscr{P}_{\omega}(\alpha)$ be functions such that $f\left(\xi_{1}\right) \cap f\left(\xi_{2}\right)=\varnothing$. Let, also, $\left\{W_{1}, W_{2}, \ldots, W_{n}, \ldots: n<\omega\right\}$ be a partition of $\alpha$ into pairwise disjoint sets with $\left|W_{n}\right|=\alpha$ for all $n<\omega$.

Then, there is an $A \subset \alpha$, with $\left|W_{n} \cap A\right|=\alpha$, such that if $\xi_{1}, \xi_{2} \in A, \xi_{1} \neq \xi_{2}$, then $f\left(\xi_{1}\right) \cap \phi\left(\xi_{2}\right)=\varnothing$.

2.

2.1. Definition. Let $I$ be a nonempty set and $\xi \in I$.

By $\Pi_{\xi}:\{-1,1\}^{I} \rightarrow\{-1,1\}$ we denote the projection onto the $\xi$ th coordinate.

Let $M$ be a finite subset of $I$; we let $\Pi_{M}:\{-1,1\}^{I} \rightarrow\{-1,1\}$ be the function

$$
\begin{gathered}
\prod_{i=1}^{n} \Pi_{\xi_{1}} \text { if } M \neq \varnothing \text { and } M=\left\{\xi_{1}, \xi_{2}, \ldots, \xi_{n}\right\}, \text { and } \\
\Pi_{M}(x)=1 \text { for all } x \in\{-1,1\}^{I} \text { if } M=\varnothing .
\end{gathered}
$$

It is easy to prove the following.

2.2. Lemma. Let $I$ be a nonempty set. Then the set $\left\{\Pi_{M}: M \in \mathscr{P}_{\omega}(I)\right\}$ generates a linear space dense in $L^{1}\{-1,1\}^{I}$.

Let $f$ be a function on $\{-1,1\}^{I}$ and $N \subset I$. We say that $f$ depends on the set $N$ if for all elements $y_{1}, y_{2}$ of $\{-1,1\}^{I N}$ and $x \in\{-1,1\}^{N}$ it holds that $f\left(x, y_{1}\right)=$ $f\left(x, y_{2}\right)$. 
If $f$ is an essentially bounded measurable complex function on $\{-1,1\}^{I}$, there is an $h$ that differs from $f$ on a set of null measure and $h$ depends on a countable subset of $I$.

In the remainder, by writing $f \in L^{\infty}\{-1,1\}^{I}$ we mean that $[f] \in L^{\infty}\{-1,1\}^{I}$, and $f$ depends on a countable subset of $I$.

2.3. Definition. Let $I$ be a set and $K$ a nonempty subset of $I$. We let

$$
P_{K}: L^{1}\{-1,1\}^{K} \rightarrow L^{1}\{-1,1\}^{I}
$$

denote the natural embedding, and

$$
P_{K}^{*}: L^{\infty}\{-1,1\}^{I} \rightarrow L^{\infty}\{-1,1\}^{K}
$$

be the conjugate of $P_{K}$.

Also, by $L^{\infty}\{-1,1\}$ we denote $R$ and by $P^{*}$ the integration functional.

2.4. LeMma. Let $I$ be a set, $K$ a subset of $I$ and $f$ an element of $L^{\infty}\{-1,1\}^{I}$ which depends on a set $N$ of coordinates. Then

$$
P_{K}^{*}(f)=P_{K \cap N}^{*}(f)
$$

where we consider $P_{K \cap N}^{*}(f)$ as an element of $L^{\infty}\{-1,1\}^{K}$ in the natural way.

Proof. We set $f_{1}=P_{K}^{*}(f)$ and $f_{2}=P_{K \cap N}^{*}(f)$. From Lemma 2.2 it is enough to check the equality of $f_{1}, f_{2}$ on the set $\left\{\Pi_{M}: M \in \mathscr{P}_{\omega}(K)\right\}$.

Case 1. $M \subset K \cap N$. Then $f_{2}\left(\Pi_{M}\right)=\int P_{K \cap N}\left(\Pi_{M}\right) f d \mu=\int \Pi_{M} f d \mu=f_{1}\left(\Pi_{M}\right)$.

Case 2. $M \nsubseteq K \cap N$. Then there is $\xi \in M$ and $\xi \notin K \cap N$. Then $f_{2}\left(\Pi_{M}\right)=$ $\int f_{2} \Pi_{M} d \mu=\int f_{2} \Pi_{M \backslash\{\xi\}} \Pi_{\xi} d \mu=\int \Pi_{\xi} d \mu \int f_{2} \Pi_{M \backslash\{\xi\}} d \mu=0$. On the other hand

$$
f_{1}\left(\Pi_{M}\right)=\int f P_{K}\left(\Pi_{M}\right) d \mu=\int f \Pi_{M} d \mu=0 .
$$

So the proof is complete.

2.5. Corollary. Let $K \subset I$ and $f \in L^{\infty}\{-1,1\}^{I}$, which depends on a set $N$ of coordinates; then $P_{K}^{*}(f)$ depends on a subset of $K \cap N$.

3. In this section we establish the possibility of embedding $l_{\alpha}^{1}$ isomorphically into a Banach space $X$, assuming that its dual $X^{*}$ contains isomorphically $L^{1}\{-1,1\}^{\alpha}$, provided $\alpha>\omega^{+}$.

Pełczynski has proved this statement for $\alpha=\omega$ and conjectured in [13] that this statement is true for all cardinals $\alpha$. Thus in Theorem 3.1 below we prove this conjecture for cardinals greater than $\omega^{+}$.

In Theorem 3.2, we prove that for certain classes of cardinals, the conclusion of Theorem 3.1 also holds under weaker assumptions.

3.1. TheOREM. Let $\alpha$ be a cardinal greater than $\omega^{+}$and $X$ a Banach space such that $L^{1}\{-1,1\}^{\alpha}$ is isomorphic to a subspace of $X^{*}$. Then $X$ has a subspace isomorphic to $l_{\alpha}^{1}$.

Proof. Let $T: L^{1}\{-1,1\}^{\alpha} \rightarrow X^{*}$ be an isomorphism and we denote by

$$
T^{*}: X^{* *} \rightarrow L^{\infty}\{-1,1\}^{\alpha}
$$


the conjugate of $T$, which is onto and $w^{*}$-continuous. From Goldstine's and open mapping theorems, there is a natural number $\kappa>0$ such that $T^{*}\left(S_{x}^{\kappa}\right)$ is $w^{*}$-dense in $S_{L^{\infty}\{-1,1\}^{\alpha}}^{1}$, where $S_{x}^{\kappa}=\{x \in X:\|x\| \leqslant \kappa\}$ and $S_{L^{\infty}\{-1,1\}^{\alpha}}^{1}$ is the unit ball of $L^{\infty}\{-1,1\}^{\alpha}$.

Let $\varepsilon>0$. We choose a family $\left\{x_{\xi}: \xi<\alpha\right\}$ of elements of $S_{x}^{\kappa+\varepsilon}$ according to the rule:

For $\xi<\alpha$ we consider the projection

$$
P_{\{\xi\}}^{*}: L^{\infty}\{-1,1\}^{\alpha} \rightarrow L^{\infty}\{-1,1\}^{(\xi)}
$$

then $P_{\{\xi\}}^{*} \circ T^{*}\left(S_{x}^{\kappa+\varepsilon}\right)$ contains the unit ball of $L^{\infty}\{-1,1\}^{\{\xi\}}$, and consequently there is $x_{\xi} \in S_{x}^{\kappa+\varepsilon}$ such that $P_{\{\xi\}}^{*} \circ T^{*}\left(x_{\xi}\right)=\Pi_{\xi}$.

For every $\xi<\alpha$ we set $N_{\xi}$ to be the countable subset of $\alpha$ on which the function $T^{*}\left(x_{\xi}\right)$ depends.

We apply Hajnal's theorem for the family $\left\{N_{\xi}: \xi<\alpha\right\}$ and we define a subset $A$ of $\alpha$ such that, for every $\xi \in A, A \cap N_{\xi} \subset\{\xi\}$.

Let $P_{A}^{*}: L^{\infty}\{-1,1\}^{\alpha} \rightarrow L^{\infty}\{-1,1\}^{A}$, and $\xi \in A$. Since $P_{\{\xi\}}^{*} \circ T^{*}\left(x_{\xi}\right)=\Pi_{\xi}, T^{*}\left(x_{\xi}\right)$ depends on the $\xi$ th coordinate, and so $A \cap N_{\xi}=\{\xi\}$. Now from Lemma 2.4, we have that

$$
P_{A}^{*} \circ T^{*}\left(x_{\xi}\right)=P_{\{\xi\}}^{*} \circ T^{*}\left(x_{\xi}\right)=\Pi_{\xi} \text { for all } \xi \in A,
$$

and since $\left\{\Pi_{\xi}: \xi \in A\right\}$ is equivalent to the usual $l^{1}(A)$ base, the same is true for the family $\left\{x_{\xi}: \xi \in A\right\}$.

3.2. TheORem. Let $\alpha$ be a cardinal with $\operatorname{cf}(\alpha)>\omega$ and $\alpha>\omega^{+}$. If $T: L^{1}\{-1,1\}^{\alpha}$ $\rightarrow X^{*}$ is a bounded one-to-one linear operator then $l_{\alpha}^{1}$ is isomorphic to a subspace of $X$.

Proof. Using the same arguments as in Theorem 3.1, we can choose a set $D \subset \alpha$ and a family $\left\{x_{\xi}: \xi \in D\right\}$ of elements of $X$ such that the family $\left\{P_{D}^{*} \circ T^{*}\left(x_{\xi}\right)\right.$ : $\xi \in D\}$ is equivalent to the usual base of $l_{D}^{1}$, with $|D|=\alpha$.

Since $\operatorname{cf}(\alpha)>\omega$ we choose a set $A \subset D$ and $M>0$ such that $|A|=\alpha$ and $\left\|x_{\xi}\right\|<M$ for all $\xi \in A$.

Then the family $\left\{x_{\xi}: \xi \in A\right\}$ is equivalent to the usual base of $l^{1}(A)$.

3.3. Remark. Theorem 3.1 has been proved for regular cardinals $\alpha \gg \omega^{+}$by Haydon in [9], and for cardinals $\alpha$ such that $\alpha$ and $\operatorname{cf}(\alpha) \gg \omega^{+}$by Negrepontis and the author in [3]. These proofs use the Erdös-Rado lemma and its extension to singular cardinals given in [3].

4.

4.1. Definition. Let $\alpha$ be a cardinal. We set

$$
Z=\left(\sum_{n<\omega} \oplus l^{\infty}(n)\right)_{1} \text { and } Z_{\alpha}=\left(\sum_{\xi<\alpha} \oplus Z_{\xi}\right),
$$

where $Z_{\xi}=Z$ for all $\xi<\alpha$.

REMARK. The space $Z_{\alpha}$ has been introduced in [7] by Hagler and Stegall; it is useful in the study of dual spaces; in fact, Stegall has proved in [18] that an 
isomorphic embedding of $Z_{\alpha}$ into a Banach space $X$ implies an isomorphism between $\left(C\{-1,1\}^{\alpha}\right)^{*}$ and a complemented subspace of $X^{*}$.

In this section we study the embedding of $Z_{\alpha}$ into a Banach spaces $X$ for which $X^{*}$ has a relationship with $L^{1}(\lambda)$ spaces.

4.2. THEOREM. Let $\alpha$ be a cardinal greater than $\omega^{+}, X$ a Banach space and $T, U$ operators such that $T: L^{1}\{-1,1\}^{\alpha} \rightarrow X^{*}$ and $U: X^{*} \rightarrow L^{1}(\lambda)$ for some measure $\lambda$ such that $U \circ T: L^{1}\{-1,1\}^{\alpha} \rightarrow L^{1}(\lambda)$ is an isomorphism. Then $X$ has a subspace isomorphic to $Z_{\alpha}$.

Proof. We denote by $U^{*}, T^{*}$ the conjugate operators of $U$ and $T$, respectively. Also, we define a well-order $\prec$ on $\alpha \times \omega$, so that this set has the same order type as the cardinal $\alpha$.

Using transfinite induction we choose

(i) a family $\left\{X_{(\xi, n)}: \xi<\alpha, n<\omega\right\}$ of finite dimensional subspaces of $X$;

(ii) for every $(\xi, n) \in \alpha \times \omega$ a family $\left\{g_{(\xi, j)}: 1 \leqslant j \leqslant 2 n\right\}$ of elements of $T\left(L^{1}\{-1,1\}^{\alpha}\right)$

(iii) a family $\left\{A_{(\xi, n)}:(\xi, n) \in \alpha \times \omega\right\}$ of pairwise disjoint countable subsets of $\alpha$ such that

(a) for every $(\xi, n) \in \alpha \times \omega$ there is a family $\left\{x_{(\xi, i)}: 1 \leqslant i \leqslant n\right\}$ of elements of $X_{(\xi, n)}$ that generates $X_{(\xi, n)}$;

(b) there are positive numbers $\vartheta_{1}, \vartheta_{2}$ such that for every $(\xi, n) \in \alpha \times \omega$ and $x \in X_{(\xi, n)}$ where $x=\sum_{i=1}^{n} t_{i} x_{(\xi, i)}$ we have that

$$
\vartheta_{1} \max \left|t_{i}\right| \leqslant\|x\| \leqslant \vartheta_{2} \max \left|t_{i}\right|,
$$

and consequently there is $\Lambda>0$ such that the Banach-Mazur distance $d\left(X_{(\xi, n)}, l^{\infty}(n)\right) \leqslant \Lambda ;$ and

(c) for every $(\xi, n) \in \alpha \times \omega$ and $1 \leqslant j \leqslant 2 n$ there is $\phi_{(\xi, j)} \in L^{1}\{-1,1\}^{\alpha}$ depending on the set $A_{(\xi, n)}$ such that

(c) $T \phi_{\left(\xi_{j}\right)}=g_{\left(\xi_{j}\right)},\left\|\phi_{\left(\xi_{j}\right)}\right\|=1$, and

$\left(c_{2}\right)$ there is $\vartheta>0$ and $\varepsilon>0$ such that

$$
\begin{aligned}
& g_{(\xi, j)}\left(x_{(\xi, i)}\right) \geqslant \theta \quad \text { if } j=i, \\
& g_{(\xi, j)}\left(x_{(\xi, i)}\right) \leqslant-\theta \quad \text { if } j=n+i, \text { and } \\
& \mid g_{(\xi, j)}\left(x_{(\xi, i)} \mid \leqslant \varepsilon / n \quad\right. \text { otherwise. }
\end{aligned}
$$

Let $\left(\xi_{0}, n_{0}\right) \in \alpha \times \omega$ and we suppose inductively that for each $(\xi, n) \prec\left(\xi_{0}, n_{0}\right)$ we have chosen $X_{(\xi, n)},\left\{g_{(\xi, j)}: 1 \leqslant j \leqslant 2 n\right\}, A_{(\xi, n)}$ so that they satisfy conditions (a), (b), (c) above.

For $(\xi, n) \prec\left(\xi_{0}, n_{0}\right)$ we let $N_{(\xi, n)}$ denote the countable set of coordinates on which the finite dimensional subspace $T^{*}\left(X_{(\xi, n)}\right)$ of $L^{\infty}\{-1,1\}^{\alpha}$ depends.

Since the cardinality of the set

$$
H_{\left(\xi_{0}, n_{0}\right)}=\left(\bigcup_{(\xi, n)<\left(\xi_{0}, n_{0}\right)} N_{(\xi, n)}\right) \cup\left(\bigcup_{(\xi, n)<\left(\xi_{0}, n_{0}\right)} A_{(\xi, n)}\right)
$$

is less than $\prec$, there is an infinite countable set $A_{\left(\xi_{0}, n_{0}\right)} \subset \boldsymbol{\alpha} \backslash H_{\left(\xi_{0}, n_{0}\right)}$. 
We choose a sequence $\left\{O_{\left(\xi_{0}, \lambda\right)}: \lambda<\omega\right\}$ of measurable subsets of $\{-1,1\}^{\alpha}$ such that

(i) each $O_{\left(\xi_{0}, \lambda\right)}$ depends on $A_{\left(\xi_{0}, n_{0}\right)}$ and $\mu\left(O_{\left(\xi_{0}, \lambda\right)}\right)>0$; and

(ii) $O_{\left(\xi_{0}, \lambda_{1}\right)} \cap O_{\left(\xi_{0}, \lambda_{2}\right)}=\varnothing$ for all $\lambda_{1}<\lambda_{2}<\omega$.

We set

$$
\phi\left(\xi_{0}, \lambda\right)=\mu\left(O_{\xi_{0} \lambda}\right)^{-1} \cdot \chi O_{\left(\xi_{0} \lambda\right)},
$$

and let $f_{\left(\xi_{0} \lambda\right)}=U \circ T\left(\phi_{\left(\xi_{0}, \lambda\right)}\right)$ for all $\lambda<\omega$.

Since $\left\{\phi_{\left(\xi_{0}, \lambda\right)}: \lambda<\omega\right\}$ is equivalent to the canonical base of $l_{\omega}^{1}$, and $U \circ T$ is an isomorphism, it follows that there are positive numbers $m_{1}, m_{2}$ that depend only on the operators $(U, T)$ such that, for each choice of scalars $\left\{c_{1}, c_{2}, \ldots, c_{\kappa}\right\}$ and $\left\{\lambda_{1}, \lambda_{2}, \ldots, \lambda_{\kappa}\right\}$, we have

$$
m_{1} \sum_{i=1}^{\kappa}\left|c_{i}\right| \geqslant\left\|\sum_{i=1}^{\kappa} c_{i} f_{\left(\xi_{0}, \lambda_{i}\right)}\right\| \geqslant m_{2} \sum_{i=1}^{\kappa}\left|c_{i}\right|
$$

From Dor's theorem [5, Theorem B], it follows that there is a sequence $\left\{\mathcal{Q}_{\left(\xi_{0}, \lambda\right)}\right.$ : $\lambda<\omega\}$ of pairwise disjoint $\lambda$-measurable sets such that

$$
\int_{\mathscr{Q}_{\left(\xi_{0} \lambda\right)}}\left|f_{\left(\xi_{0} \lambda\right)}\right| d \mu \geqslant \frac{m_{2}^{2}}{m_{1}} .
$$

Also from Rosenthal's lemma [15] and for $\varepsilon<m_{2}^{2} / 16 m_{1}$ there is a subsequence $\left\{\mathcal{Q}_{\left(\xi_{0}, \lambda_{k}\right)}: \kappa<\omega\right\}$ such that

$$
\int_{\cup_{i \neq k} \mathcal{Q}_{\left(\xi_{0}, \lambda_{1}\right)}}\left|f_{\left(\xi_{0}, \lambda_{x}\right)}\right| d \mu<\frac{\varepsilon}{n_{0}} .
$$

We choose $\left\{w_{\left(\xi_{0} \lambda \lambda^{\prime}\right.}: \kappa<\omega\right\}$ a sequence of elements of $L^{\infty}\{-1,1\}^{\alpha}$ such that

(i) $\left\|w_{\left(\xi_{0} \lambda_{k}\right)}\right\|_{\infty}=1$,

(ii) $\operatorname{supp} w_{\left(\xi_{0}, \lambda_{1}\right)} \subset \mathcal{Q}_{\left(\xi_{0} \lambda_{\lambda}\right)}$, and

(iii) $\int f_{\left(\xi_{0}, \lambda_{k}\right)} \cdot w_{\left(\xi_{0}, \lambda_{k}\right)} d \mu>m_{2}^{2} / 2 m_{1}$.

We choose $2 n_{0}$ elements of the sequence $\left\{\lambda_{\kappa}: \kappa<\omega\right\}$, say $\left\{\lambda_{\kappa_{1}}, \ldots, \lambda_{\kappa_{2 n_{0}}}\right\}$, and we set

$$
x_{\left(\xi_{0}, i\right)}^{* *}=U^{*} w_{\left(\xi_{0}, \lambda_{i}\right)}-U^{*} w_{\left(\xi_{0}, \lambda_{m_{0}+1}\right)} .
$$

It is easy to verify that if $\left\{c_{1}, c_{2}, \ldots, c_{n_{0}}\right\}$ are scalars, then

$$
\left\|U^{*}\right\| \max _{1<i<n_{0}}\left|c_{i}\right| \geqslant\left\|\sum c_{i} x_{\left(\xi_{0}, i\right)}^{* *}\right\| \geqslant \frac{m_{2}^{2}}{4 m_{1}} \max _{1<i<n_{0}}\left|c_{i}\right| .
$$

Also we set $\phi_{\left(\xi_{0 j}\right)}=\phi_{\left(\xi_{0}, \lambda k_{j}\right)}$ for all $1 \leqslant j \leqslant 2 n_{0}$, and $g_{\left(\xi_{0 j}\right)}=T\left(\phi_{\left(\xi_{0}, j\right)}\right)$ for all $1<j<2 n_{0}$, and we observe that

$$
\begin{aligned}
& g_{\left(\xi_{\xi_{0}}, i\right)}\left(x_{\left(\xi_{0}, i\right)}^{* *}\right)=\int w_{\left(\xi_{0}, i\right)} \cdot f_{\left(\xi_{0}, i\right)} d \mu-\int w_{\left(\xi_{0}, n_{0}+i\right)} f_{\left(\xi_{0}, i\right)} d \mu \\
& \geqslant m_{2}^{2} / 2 m_{1}-m_{2}^{2} / 8 m_{1} \geq m_{2}^{2} / 4 m_{1}=\vartheta, \\
& g_{\left(\xi_{0}, n_{0}+i\right)}\left(x_{\left(\xi_{0}, i\right)}^{* *}\right) \leqslant-m_{2}^{2} / 4 m_{1}=\vartheta, \text { and }\left|g_{\left(\xi_{\sigma_{0}}\right)}\left(x_{\left(\xi_{0}, i\right)}^{* *}\right)\right|<\varepsilon / n_{0} .
\end{aligned}
$$


From the local reflexivity theorem [11] we choose a family $\left\{x_{\left(\xi_{0}, i\right)}: 1 \leqslant i \leqslant n_{0}\right\}$ of elements of $X$ such that

(i) $g_{\left(\xi_{0} j\right)}\left(x_{\left(\xi_{0}, i\right)}\right)=x_{\left(\xi_{0}, i\right)}^{* *}\left(g_{\left(\xi_{0} j\right)}\right)$ for all $1 \leqslant i \leqslant n_{0}$ and $1 \leqslant j \leqslant 2 n_{0}$,

(ii) if $x=\sum_{i=1}^{n_{0}} c_{i} x_{\left(\xi_{0}, i\right)}$ then

$$
\vartheta_{1} \max \left|c_{i}\right|>\|x\| \geqslant \vartheta_{2} \max \left|c_{i}\right|,
$$

where $\vartheta_{1}=\left\|U^{*}\right\|+\varepsilon, \vartheta_{2}=m_{2}^{2} / 4 m_{1}-\varepsilon \geqslant m_{2}^{2} / 8 m_{1}$, and we set $X_{\left(\xi_{0}, n_{0}\right)}$ for the space generated by the family $\left\{x_{\left(\xi_{0}, i\right)}: 1 \leqslant i \leqslant n_{0}\right\}$. This is the end of the inductive construction.

For each $(\xi, n)$ we let $N_{(\xi, n)}$ be the countable subset of $\alpha$ on which the finite dimensional space $T^{*}\left(X_{(\xi, n)}\right)$ depends. We also set $A_{n}=\{(\xi, n): \xi<\alpha\}$.

We apply Proposition 1.4 for the families $\left\{N_{(\xi, n)}:(\xi, n) \in \alpha \times \omega\right\},\left\{A_{(\xi, n)}\right.$ : $(\xi, n) \in \alpha \times \omega\}$ and the partition $\left\{\Delta_{n}: n<\omega\right\}$, and we choose $\Delta \subset \alpha \times \omega$ such that $\left|\Delta \cap \Delta_{n}\right|=\alpha$, and for every $\left(\xi_{1}, n_{1}\right) \neq\left(\xi_{2}, n_{2}\right)$ elements of $\Delta$ we have that $N_{\left(\xi_{1}, n_{1}\right)} \cap A_{\left(\xi_{2}, n_{2}\right)}=\varnothing$. We claim that the space that generates the family $\left\{X_{(\xi, n)}\right.$ : $(\xi, n) \in \Delta\}$ is isomorphic to $Z_{\alpha}$.

In order to prove this, it is enough to verify that if $\left(\xi_{1}, n_{1}\right), \ldots,\left(\xi_{k}, n_{k}\right)$ is a finite choice of pairwise different elements of $\Delta$, and $x_{1} \in X_{\left(\xi_{1}, n_{1}\right)}, \ldots, x_{\kappa} \in X_{\left(\xi, n_{k}\right)}$ with $x_{j}=\sum_{i=1}^{n_{j}} t_{i}^{j} x_{(\xi, i)}$ for all $1 \leqslant j \leqslant \kappa$, then

$$
\frac{\vartheta}{4}\left\|T^{*}\right\|^{-1} \sum_{j=1}^{n} \max _{1<i<n_{j}}\left|t_{i}^{j}\right| \leqslant\left\|\sum_{j=1}^{\kappa} x_{j}\right\| \leqslant \vartheta_{2} \sum_{j=1}^{\kappa} \max _{1<i<n_{j}}\left|t_{i}^{j}\right| .
$$

We set $D=\cup\left\{A_{(\xi, n)}:(\xi, n) \in \Delta\right\}$. Let $(\xi, n) \in \Delta$, and $x \in X_{(\xi, n)}$ with $x \neq 0$; then $x$ has the form

$$
x=\sum_{i=1}^{n} t_{i} x_{(\xi, i)} .
$$

We consider the element $P_{D}^{*} \circ T^{*}(x)$ of $L^{\infty}\{-1,1\}^{D}$. Since $D \cap N_{(\xi, n)} \subset A_{(\xi, n)}$ we have, from Corollary 2.5 , that $P_{D}^{*} \circ T^{*}(x)=P_{A_{(\xi, n)}^{*}}^{*} \circ T^{*}(x)$, and consequently this element depends on the set $A_{(\xi, n)}$.

Let $i_{0} \in\{1, \ldots, n\}$ be such that $\left|t_{i_{0}}\right|=\max \left\{\left|t_{i}\right|: 1 \leqslant i \leqslant n\right\}$, and we set

$$
A_{i_{0}}=\left\{z \in\{-1,1\}^{D}: P_{D}^{*} \circ T^{*}(x)(z) \geqslant\left|t_{i_{0}}\right| \vartheta / 4\right\} .
$$

Then

$$
\mu_{D}\left(A_{i_{0}}\right)>0
$$

Assume first that $t_{i_{0}}>0$; then

$$
T^{*}(x)\left(\phi_{\left(\xi, i_{0}\right)}\right)=x\left(g_{\left(\xi, i_{0}\right)}\right) \geqslant t_{i_{0}} \cdot \vartheta-\left|\sum_{\substack{j=1 \\ j \neq i_{0}}}^{n} t_{j} \frac{\varepsilon}{n}\right| \geqslant t_{i_{0}} \frac{\vartheta}{2} .
$$

In the case $t_{i_{0}}<0$, with similar arguments we find that $T^{*}(x)\left(\phi_{\left(\xi, n+i_{0}\right)}\right) \leqslant-t_{i_{0}}$. $\vartheta / 2$, and the fact that the functions $\phi_{\left(\xi, i_{0}\right)}, \phi_{\left(\xi, n+i_{0}\right)}$ are positive elements of $L^{1}\{-1,1\}^{D}$ of norm one implies that $(*)$ holds. 
Now if $\left(\xi_{1}, n_{1}\right) \neq\left(\xi_{2}, n_{2}\right) \neq \cdots \neq\left(\xi_{\kappa}, n_{\kappa}\right)$ is a finite choice, and $x_{j} \in X_{\left(\xi, \eta_{j}\right)}$ is a nonzero element of the form

$$
x_{j}=\sum_{i=1}^{n_{j}} t_{i}^{j} x_{(\xi, i)} \text { for all } j=1, \ldots, \kappa
$$

and $\left|t_{i j}\right|=\max _{1<i \leqslant n_{j}}\left|t_{i}^{j}\right|$, then the family $\left\{A_{i_{j}}: 1 \leqslant j \leqslant \kappa\right\}$ is stochastically independent. So if $K=\bigcap_{j=1}^{\kappa} A_{i}$, then $\mu(K)>0$ and for the element $h=\mu_{D}(K)^{-1} \cdot \chi_{K} \in$ $L^{1}\{-1,1\}^{\alpha}$ we have that $\|h\|_{1}=1$, and

$$
\begin{aligned}
\left\|\sum_{j=1}^{\kappa} \lambda_{j}\right\| & \geqslant\left\|T^{*}\right\|^{-1} \cdot\left\|\sum_{j=1}^{\kappa} P_{D}^{*} \circ T^{*}\left(x_{j}\right)\right\| \\
& \geqslant\left\|T^{*}\right\|^{-1} \cdot\left|\sum_{j=1}^{\kappa} P_{D}^{*} \circ T^{*}\left(x_{j}\right)(h)\right| \\
& =\frac{1}{\mu_{D}(K)}\left\|T^{*}\right\|^{-1} \cdot \sum_{j=1}^{\kappa} \int_{\kappa} P_{D}^{*} \circ T^{*}\left(x_{j}\right) d \mu \geqslant\left\|T^{*}\right\|^{-1} \frac{\vartheta}{2} \sum_{j=1}^{\kappa}\left|t_{i j}\right| .
\end{aligned}
$$

On the other hand $\left\|\sum_{j=1}^{\kappa} x_{j}\right\| \leqslant \sum_{j=1}^{\kappa}\left\|x_{j}\right\|<\vartheta_{2} \sum_{j=1}^{\kappa}\left|t_{i j}\right|$. On the other hand $\left\|\sum_{j=1}^{\kappa} x_{j}\right\| \leqslant \sum_{j=1}^{\kappa}\left\|x_{j}\right\| \leqslant \vartheta_{2} \sum_{j=1}^{\kappa}\left|t_{i}\right|$, and the proof is complete.

4.3. REMARK. The statement of Theorem 4.2 is valid in the case $\alpha=\omega$ as well. In fact, it follows from a result of Starbird in [17], since $U \circ T: L^{1}(0,1) \rightarrow L^{1}(\lambda)$ is an isomorphism, that there is a subspace $W$ isomorphic to $L^{1}(0,1)$ such that $U \circ T(W)$ is complemented into $L^{1}(\lambda)$. So $X^{*}$ contains a complemented subspace isomorphic to $L^{1}(0,1)$, and it follows from [7] that $X$ contains the space $Z$.

4.4. Corollary. Let $\alpha$ be a cardinal greater than $\omega^{+}$and $X$ a Banach space such that $L^{1}\{-1,1\}^{\alpha}$ is isomorphic to a complemented subspace of $X^{*}$. Then $Z_{\alpha}$ is isomorphic to a subspace of $X$.

4.5. Corollary. Let $X$ be an $\mathfrak{L}_{\infty}$ space such that $L^{1}\{-1,1\}^{\alpha}$ is isomorphic to a subspace of $X^{*}$. Then $X$ contains a subspace isomorphic to $Z_{\alpha}$ and $\left(\Sigma_{2^{\alpha}} \oplus L^{1}\{-1,1\}^{\alpha}\right)_{1}$ is isomorphic to a complemented subspace of $X^{*}$.

Proof. Since $X$ is an $\mathfrak{L}_{\infty}$ space, $X^{*}$ is a complemented subspace of $L^{1}(\lambda)$ for some measure $\lambda$; from Theorem 4.2 we have that $Z_{\alpha}$ is isomorphic to a subspace of $X$. The second part is a consequence of a result of Stegall in [18].

5. In this section we investigate universal embeddings of $l_{\alpha}^{1}$ into subspaces $X$ of $L^{\infty}\{-1,1\}^{\alpha}$ with $\operatorname{dim} X=\alpha$, under conditions that depend only on the form of the cardinal $\alpha$ (Theorems 5.1, 5.2).

5.1. TheOREM. Let $\alpha$ be a cardinal such that $\alpha$ is strongly $\omega^{+}$-inaccessible and $\operatorname{cf}(\alpha)>\omega^{+}$. Let, also, $\left\{x_{\xi}: \xi<\alpha\right\}$ be a family of elements of $L^{\infty}\{-1,1\}^{\alpha}$ with $\left\|x_{\xi}\right\| \leqslant M$ and $\left\|x_{\xi}-x_{\xi}\right\|>\theta>0$ for all $\xi, \zeta \in \alpha, \xi \neq \zeta$. Then there is an $A \subset \alpha$, with $|A|=\alpha$ and a family $\left\{y_{\eta}: \eta \in A\right\}$ of elements of $L^{\infty}\{-1,1\}^{\alpha}$ such that

(i) for each $n \in A$ there are $\xi_{(\eta, 1)}, \xi_{(\eta, 2)}$ such that $y_{\eta}=x_{\xi_{(\eta, 1)}}-x_{\xi_{(\eta, 2)}}$; 
(ii) if $c_{1}, c_{2}, \ldots, c_{\kappa}$ are complex numbers and $\eta_{1} \neq \eta_{2} \neq \cdots \neq \eta_{\kappa}$ are elements of $A$, then

$$
2 M \sum_{i=1}^{\kappa}\left|c_{i}\right| \geqslant\left\|\sum_{i=1}^{\kappa} c_{i} y_{\eta_{n}}\right\| \geqslant \frac{\theta}{172} \sum_{i=1}^{\kappa}\left|c_{i}\right|
$$

Proof. Using transfinite induction, we choose a family $\left\{y_{\eta}: \eta<\alpha\right\}$ in the following way:

Let $\eta_{0}<\alpha$ be an ordinal and assume that, for every $\eta<\eta_{0}, y_{\eta}$ has been chosen.

Let $N_{\eta}$ be the subset of $\alpha$ on which the function $y_{\eta}$ depends, and we set $I_{\eta_{0}}=\cup_{\eta<\eta_{0}} N_{\eta}$. Since $\alpha \gg \omega^{+}$we have that

$$
\operatorname{dim} L^{\infty}\{-1,1\}^{I_{n_{0}}}<\left|L^{\infty}\{-1,1\}^{I_{n_{0}}}\right|=\left|I_{\eta_{0}}\right|^{\omega}<\alpha .
$$

Consequently, there are two elements of $\left\{x_{\xi}: \xi<\alpha\right\}$ such that

$$
P_{I_{\eta_{0}}}\left(x_{\xi\left(\eta_{0,1}\right)}\right)=P_{I_{\eta_{0}}}\left(x_{\xi\left(\eta_{0,2}\right)}\right) \text {. }
$$

We set $y_{\eta_{0}}=x_{\xi_{\left(\eta_{0}\right)}}-x_{\xi_{\left(\eta_{0}\right)}}$. This completes the definition of the family $\left\{y_{\eta}\right.$ : $\eta<\alpha\}$. We observe that

(i) $\int y_{\eta} d \mu=0$;

(ii) for each $\eta<\alpha$ there is a finite $A_{\eta}$ subset of $\alpha$, and a function $g_{\eta}=r_{i}^{\eta} \Pi_{M_{\eta}}$ $+\cdots+r_{k}^{\eta} \Pi_{M_{\eta_{k}}}$, where $A_{\eta} \subset N_{\eta}, M_{\eta_{i}} \subset A_{\eta}, M_{\eta_{i}} \backslash I_{\eta} \neq \varnothing\left(I_{\eta}=\cup_{\eta^{\prime}<\eta} N_{\eta^{\prime}}\right)$ and $\left\|g_{\eta}\right\|_{1} \leqslant 2,\left|\int y_{\eta} \cdot g_{\eta} d \mu\right|>\theta / 2$.

Property (i) is obvious from the inductive choice of $\left\{y_{\eta}: \eta<\alpha\right\}$. For (ii), we choose $A_{\eta} \subset N_{\eta}$ finite and $h_{\eta} \in L^{1}\{-1,1\}^{A_{\eta}}$, such that

(a) $\left\|h_{\eta}\right\|_{1}=1$

(b) $\int y_{\eta} \cdot h_{\eta} d \mu>\theta / 2$.

Let $h_{\eta}=r_{1}^{\eta} \Pi_{M_{\eta_{1}}}+\cdots+r_{\lambda}^{\eta} \Pi_{M_{\eta_{\lambda}}}$ be an expression of $h_{\eta}$ with the function $\left\{\Pi_{M}\right.$ : $\left.M \subset A_{\eta}\right\}$. We decompose the family $L=\left\{M_{\eta_{1}}, \ldots, M_{\eta_{\lambda}}\right\}$ into two sets, namely

$$
L_{1}=\left\{M_{\eta_{1}}: M_{\eta_{1}} \subset I_{\eta}\right\} \text { and } L_{2}=L \backslash L_{1} \text {. }
$$

Since $P_{I_{\eta}}^{*}\left(y_{\eta}\right)=0$, it follows that $\int y_{\eta} \Pi_{M_{\eta_{i}}} d \mu=0$ for all $M_{\eta_{i}} \in L_{1}$. So

$$
\frac{\theta}{2}<\left|\int y_{\eta} \cdot h_{\eta} d \mu\right|=\left|\Sigma_{M_{\eta_{i}} \in L_{2}} \cdot r_{i}^{\eta_{i}} h_{\eta} \Pi_{M_{\eta_{i}}} d \mu\right| \text {. }
$$

We put $g_{\eta}=\Sigma_{M_{\eta_{i}} \in L_{2}} \cdot r_{i}^{n} \Pi_{M_{\eta_{i}}}$, and we observe that $\left\|g_{\eta}\right\|_{1} \leqslant\left\|I \backslash P_{I_{\eta}}\right\| \cdot\left\|h_{\eta}\right\| \leqslant 2$, where $P_{I_{\eta}}$ is the usual projection of $L^{1}\{-1,1\}^{\alpha}$ onto $L^{1}\{-1,1\}^{I_{\eta}}$, and $I$ is the identity map of $L^{1}\{-1,1\}^{\alpha}$.

Now using Proposition 1.6 for the families $\left\{N_{\eta}: \eta<\alpha\right\},\left\{A_{\eta}: \eta<\alpha\right\}$ we choose a family $\left\{\Delta_{\sigma}: \sigma<\operatorname{cf}(\alpha)\right\}$ and $\left\{M_{\sigma}: \sigma<\operatorname{cf}(\alpha)\right\}, M$, such that

(i) if $\Delta=\cup_{\sigma<\operatorname{cf}(\alpha)} \Delta_{\sigma}$ then $|\Delta|=\alpha$;

(ii) $\left|M_{\sigma}\right|<\omega,|M|<\omega$; and

(iii) if $\eta_{1} \neq \eta_{2} \in \Delta_{\sigma}$, then $N_{\eta_{1}} \cap A_{\eta_{2}} \subset M_{\sigma}$; if $\sigma_{1}<\sigma_{2}$ and $\eta_{1} \in \Delta_{\sigma_{1}}, \eta_{2} \in \Delta_{\sigma_{2}}$ then $N_{\eta_{1}} \cap A_{\eta_{2}} \subset M$.

We put $D=\cup_{\eta \in \Delta} A_{\eta}$, and we consider the projection $P_{D}^{*}: L^{\infty}\{-1,1\}^{\alpha} \rightarrow$ $L^{\infty}\{-1,1\}^{D}$. From the choice of $D$ and Lemma 2.4, we have that, for each $\eta \in \Delta$, 
$P_{D}^{*}\left(y_{\eta}\right)$ depends on the set $A_{\eta}$ and so $P_{D}^{*}\left(y_{\eta}\right)$ belongs to $C\{-1,1\}^{D}$. Also, if $\eta_{1}<\eta_{2}$ are elements of $\Delta$, then

$$
\begin{aligned}
\left\|P_{D}^{*}\left(y_{\eta_{1}}\right)-P_{D}^{*}\left(y_{\eta_{2}}\right)\right\| & \geqslant \frac{1}{2}\left|\int y_{\eta_{1}} \cdot g_{\eta_{1}}-\int y_{\eta_{2}} \cdot g_{\eta_{1}}\right| \\
& =\frac{1}{2}\left|\int y_{\eta_{1}} \cdot g_{\eta_{1}}\right| \geqslant \frac{\theta}{4} .
\end{aligned}
$$

From Theorem 3.10 of [3] there is a set $A^{\prime} \subset \Delta$, with $\left|A^{\prime}\right|=\alpha$, such that if $c_{1}, c_{2}, \ldots, c_{\kappa}$ are scalars and $\eta_{1} \neq \eta_{2} \neq \cdots \neq \eta_{\kappa}$ are elements of $A^{\prime}$, then

$$
2 M \sum_{i=1}^{\kappa}\left|c_{i}\right| \geqslant\left\|\sum_{i=1}^{\kappa} c_{i} P_{D}^{*}\left(y_{\eta_{i}}\right)\right\| \geqslant \frac{\theta}{172} \sum_{i=1}^{\kappa}\left|c_{i}\right| .
$$

Thus

$$
2 M \sum_{i=1}^{\kappa}\left|c_{i}\right| \geqslant\left\|\sum_{i=1}^{\kappa} c_{i} y_{n_{n}}\right\| \geqslant \frac{\theta}{172} \sum_{i=1}^{\kappa}\left|c_{i}\right| .
$$

An immediate consequence of the above theorem is the following result.

5.2. THEOREM. Let $\alpha$ be a cardinal strongly $\omega^{+}$-inaccessible and $\operatorname{cf}(\alpha)>\omega^{+}$. Then every subspace $X$ of $L^{\infty}\{-1,1\}^{\alpha}$ with $\operatorname{dim} X=\alpha$ has a subspace isomorphic to $l_{\alpha}^{1}$.

5.3. RemarK. If we assume G.C.H. the result of Theorem 5.1 or 5.2 takes the following simple form:

If $\alpha$ is a cardinal with $\operatorname{cf}(\alpha)>\omega^{+}$and $\alpha$ is not the successor of a cardinal $\beta$ with countable cofinality, then each closed subspace $X$ of $L^{\infty}\{-1,1\}^{\alpha}$ with $\operatorname{dim} X=\alpha$ contains isomorphically a copy of $l_{\alpha}^{1}$.

It is known that if $\operatorname{cf}(\alpha)=\omega$ then there is no universal embedding of $l_{\alpha}^{1}$ into $L^{\infty}\{-1,1\}^{\alpha}$. Also, if $\alpha=\beta^{+}$and $\beta>\operatorname{cf}(\beta)=\omega$ then a subspace $X$ of $L^{\infty}\{-1,1\}^{\alpha}$ contains isomorphically a copy of $l_{\beta}^{1}$.

5.4. ReMARK. Theorems 5.1, 5.2 were proved by Negrepontis and the author in [3] for certain classes of cardinals. In particular, Theorem 4.13 of [3] states that for cardinals $\alpha$ with $\alpha, \operatorname{cf}(\alpha)$ both strongly $\omega^{+}$-inaccessible there is a universal embedding of $l_{\alpha}^{1}$ into $L^{\infty}\{-1,1\}^{\alpha}$.

The proof makes use of the Erdös-Rado lemma for regular cardinals, and its extension to singular cardinals given in [3].

5.5. AN EXAMPLE. The results of [3], like those of [6,9], and [10] depended on the existence of independent families of sets in order to get embedding of $l^{1}(\Gamma)$ spaces. The following example shows that such techniques would not be adequate to yield the result of 5.1 .

Assume M.A. $+7 \mathrm{CH}$ and let $\alpha$ be a strongly $\omega^{+}$-inaccessible cardinal with $\operatorname{cf}(\alpha)=2^{\omega}$. Then there is a family $\left\{f_{\xi}: \xi<\alpha\right\}$ of elements of $L^{\infty}\{-1,1\}^{\alpha}$ such that $\left\{f_{\xi}: \xi<\alpha\right\}$ is equivalent to the usual base of $l_{\alpha}^{1}$ but for every $r, \delta$ with $\delta>0$ the family $\left\{\left(f_{\xi}^{-1}(-\infty, r), f_{\xi}^{-1}(r+\delta, \infty)\right): \xi<\alpha\right\}$ has no essentially independent subfamily with cardinality $\alpha$ (where a family $\left\{\left(A_{\xi}, B_{\xi}\right): \xi<\alpha\right\}$ of pairs of measurable sets is said to be essentially independent if for $\xi_{1} \neq \xi_{2} \neq \cdots \neq \xi_{\kappa} \neq n_{1} \neq \cdots \neq$ $n$ we have $\left.\mu\left(\cap A_{\xi} \cap \cap B_{n_{3}}\right)>0\right)$. 
We make use of the following.

5.6. Lemma. ${ }^{1}$ Assume M.A.+ $\rceil$ C.H. There is a family $\left\{K_{\sigma}: \sigma<2^{\omega}\right\}$ of closed subsets of $\{0,1\}^{\omega}$ with $\lambda\left(K_{\sigma}\right)>0$ for all $\sigma<2^{\omega}$, such that no subfamily with $2^{\omega}$ cardinality has nonempty intersection.

We pass now to the construction of the family $\left\{f_{\xi}: \xi<\alpha\right\}$. First we choose a family $\left\{\alpha_{\sigma}: \sigma<2^{\omega}\right\}$ such that $\alpha_{\sigma}<\alpha$ and $\sum_{\sigma<\omega^{+}} \alpha_{\sigma}=\alpha$; then using transfinite induction we choose a family $\left\{\mathscr{Q}_{\xi}: \xi<2^{\omega}\right\}$ such that

(i) if $\alpha_{\sigma} \leqslant \xi<\alpha_{\sigma+1}$, then $\mathscr{Q}_{\xi} \subset K_{\sigma} \times\{-1,1\}^{\alpha-\omega}$ with $\mu\left(\mathscr{Q}_{\xi}\right)>0$; and,

(ii) if $\xi_{1}<\xi_{2}<\alpha$ then $\mu\left(\mathcal{Q}_{\xi_{1}} \triangle \mathcal{Q}_{\xi_{2}}\right)>0$.

We consider the family $\left\{X_{\mathscr{Q}_{\xi}}: \xi<\alpha\right\}$. From Theorem 5.2 there is an $A \subset \alpha$ such that $|A|=\alpha$, and the family $\left\{X_{\mathscr{Q}_{\xi}}: \xi \in A\right\}$ is equivalent to the usual base of $l^{1}(A)$. We set $f_{\xi}=X_{\text {ข }_{\xi}}$. Then the family $\left\{f_{\xi}: \xi \in A\right\}$ has the desired properties.

6. Under Martin's axiom and the negation of the continuum hypothesis we prove the analogues of Theorems 3.1 and 4.2 for the case where $\alpha=\omega^{+}$. We give all the details of the proof for the analogue of Theorem 4.2 and a description of the simpler proof of the analogue of 3.1.

6.1. Definitions and notations. Let $(P,<)$ be a partially ordered set. A subset $D$ of $P$ is dense in $P$ if for each $x \in P$ there is $y \in D$ such that $y<x$. A finite subset $F$ of $P$ is compatible if there is a $y \in P$ such that, for each $x \in F, y<x$. A subset of $P$ is a filter if each finite subset $F$ of $\mathcal{F}$ is compatible. A partially ordered set $(P,<)$ has the countable chain condition (c.c.c.) if each family of elements of $P$ consisting of pairwise incompatible elements is countable.

6.2. Martin's AXIOM (M. A.) [16]. Let $(P,<)$ be a partially ordered c.c.c. set. If $\alpha<2^{\omega}$ is a cardinal and $\left\{\Lambda_{\xi}: \xi<\alpha\right\}$ is a family of dense subsets of $P$, there is a filter $\mathscr{F}$ such that

$$
\mathscr{F} \cap \Lambda_{\xi} \neq \varnothing \text { for all } \xi<\alpha
$$

6.3. Theorem. Assume M.A. and $\neg$ C.H. Let $X$ be a Banach space and $T, U$ be bounded linear operators such that $T: L^{1}\{-1,1\}^{\omega^{+}} \rightarrow X^{*}, U: X^{*} \rightarrow L^{1}(\lambda)$ for some measure $\lambda$, and $U \circ T$ is an isomorphic embedding. Then $Z_{\omega^{+}}$embeds isomorphically into $X$.

We need some preparation before we go into the proof of this theorem. First we assume that $\|T\|=\|U\|=1$, and we choose, fix for the rest of this section, numbers $\varepsilon>0, \theta>0$ such that

$$
\theta=\frac{1}{8}\left(\left\|(U \circ T)^{-1}\right\|^{-1}\right)^{2} \text { and } \varepsilon<\theta / 2 .
$$

By $F, G$ we denote finite subsets of $\mathscr{P}_{\omega}\left(S_{X}^{1+\varepsilon}\right)$ and by $f, g$ finite subsets of $\mathscr{P}_{\omega}\left(\omega^{+}\right)$; for simplicity we write $P_{f}^{*}$ instead of $P_{\cup f}^{*}$.

\footnotetext{
${ }^{1}$ A proof can be found in F. D. Tall's paper The countable chain condition versus separability-Application of Martin's Axiom, General Topology Appl. 4 (1974), 315-339.
} 
6.4. Definition. Let $F$ be a finite subset of $\mathscr{P}_{\omega}\left(S_{X}^{1+\varepsilon}\right)$ and $f$ a finite subset of $\mathscr{P}_{\omega}\left(\omega^{+}\right)$such that $|F|=|f|=n_{F}$, and

$$
\begin{aligned}
& F=\left\{H_{1}, \ldots, H_{n_{F}}\right\}, \quad f=\left\{h_{1}, \ldots, h_{n_{F}}\right\}, \\
& H_{i}=\left\{x_{1}^{i}, \ldots, x_{k_{i}}^{i}\right\} \quad \text { for } 1 \leqslant i \leqslant n_{F}, \\
& H_{i_{1}} \cap H_{i_{2}}=\varnothing, \quad h_{i_{1}} \cap h_{i_{2}}=\varnothing \text { for } 1 \leqslant i_{1}<i_{2} \leqslant n_{F} .
\end{aligned}
$$

We say that a pair $(F, f)$, as above, is a $\left(^{*}\right)$-pair iff for $1 \leqslant i \leqslant n_{F}, 1 \leqslant j \leqslant k_{i}$ there are sets $A_{x_{j}^{i}}^{f}, B_{x_{j}^{i}}^{f_{i}}$ both nonempty, with

(i) $P_{f}^{*} \circ T^{*} x_{i}^{j}\left(A_{x_{j}^{j}}^{f}\right) \subset[-1, \theta), P_{f}^{*} \circ T^{*} x_{i}^{j}\left(B_{x_{j}}^{f_{i}}\right) \subset(\theta, 1]$.

(ii) For each $i<n_{F}, j_{1} \neq j_{2}, j_{2} \leqslant k_{i}$,

$$
P_{f}^{*} \circ T^{*} x_{j_{1}}^{i}\left(A_{x_{2}^{i}}^{f} \cup B_{x_{j_{2}}}^{f}\right) \subset\left(-\varepsilon / k_{i}, \varepsilon / k_{i}\right) .
$$

(iii) The pair $A_{x_{j}}^{f}, B_{x_{j}}^{f}$ depends on the set of coordinates $h_{i}$.

A pair $(F, f)$ is an elementary $\left({ }^{*}\right)$-pair iff $(F, f)$ is a $\left(^{*}\right)$-pair and $F=\{H\}$, $f=\{h\}$.

We will construct a partially ordered set $(P,<)$ satisfying the c.c.c. and consisting of elements of the form $(F, f)$ each being a $\left({ }^{*}\right)$-pair, and a suitable family of dense subsets of it. For this construction we need a family of elementary $\left({ }^{*}\right)$-pairs and an extension property for a $\left({ }^{*}\right)$-pair. The following Lemmas 6.5 and 6.8 ensure these steps.

6.5. Lemma. There is a family $\left\{\left(\left\{H_{\xi}\right\},\left\{h_{\xi}\right\}\right): \xi<\omega^{+}\right\}$of elementary $\left({ }^{*}\right)$-pairs such that

(i) If $\xi<\omega^{+}$and $\xi=\sigma+\kappa$ where $\sigma$ is a limit ordinal and $\kappa$ a finite ordinal then $\left|H_{\xi}\right|=\kappa+1$.

(ii) $h_{\xi} \cap \xi=\varnothing$ and $h_{\xi} \cap h_{\zeta}=\varnothing$ for all $\xi<\zeta<\omega^{+}$.

(iii) For every $\lambda_{1}, \lambda_{2}, \ldots, \lambda_{\kappa+1}$ real numbers

$$
\left\|\sum_{i=1}^{\kappa+1} \lambda_{i} x_{i}^{\xi}\right\| \leqslant(1+\varepsilon) \sup \left|\lambda_{i}\right| .
$$

For the proof of this we need the following finite version of Hajnal's theorem.

6.6. Lemma. Let $\kappa, \lambda$ natural numbers. Then for every family $N_{1}, N_{2}, \ldots, N_{\lambda^{\kappa+1}}$ of subsets of $\left\{1, \ldots, \lambda^{\kappa+1}\right\}$ with $\left|N_{i}\right| \leqslant \kappa$ for all $1 \leqslant i \leqslant n_{\lambda^{\kappa+1}}$ there is a set $A \subset$ $\left\{1, \ldots, \lambda^{\kappa+1}\right\}$ with $|A| \geqslant \lambda$ and, for every $i \in A, A \cap N_{i} \subset\{i\}$.

Proof. Assume the contrary; then we define a decomposition $\left\{\Lambda_{1}^{1}, \ldots, \Lambda_{\lambda}^{1}\right\}$ into pairwise disjoint sets, each with cardinality $\lambda^{*}$. Since the conclusion is false, there is a $1 \leqslant i_{0} \leqslant \lambda$ such that, for each $j \in \Lambda_{i_{0}}, N_{j} \cap\left(\cup_{i \neq i_{0}} \Lambda_{i}\right) \neq \varnothing$. For the set $\Lambda_{i_{0}}^{1}$ we define again a decomposition $\left\{\Lambda_{1}^{2}, \ldots, \Lambda_{\lambda}^{2}\right\}$ into pairwise disjoint sets with $\left|\Lambda_{i}^{2}\right|=$ $\lambda^{\kappa-1}$ and we define a $\Lambda_{i_{0}}^{2}$ such that, for every $j \in \Lambda_{i_{0}}^{2}, N_{j} \cap\left(\cup_{i \neq i_{0}} \Lambda_{i}^{2}\right) \neq \varnothing$. So every set $N_{j}$ for $j \in \Lambda_{i_{0}}^{2}$ contains at least two elements. Following this process in the $\kappa+1$ step we find a $j$ such that the set $N_{j}$ contains at least $\kappa+1$ elements, a contradiction. 


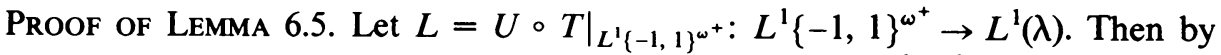
assumption, $L$ is an isomorphic embedding. We set $m=\left\|L^{1}\right\|^{-1}$. Inductively for each $\xi<\omega^{+}$with $\xi=\sigma+\kappa$ with $\sigma$ a limit ordinal and $\kappa$ a finite ordinal we set $n_{\xi}=\left(\lambda_{\kappa}\right)^{\nu_{k}}$ where $\lambda_{\kappa}=2(\kappa+1)$ and $\nu_{\kappa}=[2(\kappa+1) / \varepsilon]+1$ and we choose a finite subset of $\omega^{+}, M_{\xi}$, such that $2^{\left|M_{\xi}\right|} \geqslant n_{\xi}, M_{\xi_{1}} \cap M_{\xi_{2}}=\varnothing, M_{\xi_{1}} \cap \xi_{1}=\varnothing$ for all $\xi_{1}<\xi_{2}<\omega^{+}$.

For each $\xi<\omega^{+}$we consider elements $e_{i}^{\xi}, \ldots, e_{n_{\xi}}^{\xi}$ of $\{-1,1\}^{M_{\xi}}$ and we set for all $1 \leqslant i \leqslant n_{\xi}$

$$
g_{i}^{\xi}=\mu\left(\left\{e_{i}^{\xi}\right\}\right)^{-1} \chi_{\left\{e_{i}^{\xi}\right\}}
$$

be the norm one element of $L^{1}\{-1,1\}^{\omega^{+}}$which is defined by $l_{i}^{\xi}$. Then since $\left\|\sum_{i=1}^{n_{\xi}} \lambda_{i} g_{i}^{\xi}\right\|=\sum_{i=1}^{n_{\xi}}\left|\lambda_{i}\right|$ we have that

$$
\left\|\sum_{i=1}^{n_{\xi}} \lambda_{i} L\left(g_{i}^{\xi}\right)\right\| \geqslant m\left(\sum_{i=1}^{n_{\xi}}\left|\lambda_{i}\right|\right)
$$

and from Dor's theorem [5, Theorem B] there are $E_{1}^{\xi}, \ldots, E_{n_{\xi}}^{\xi}$ pairwise disjoint $\lambda$-measurable sets such that

$$
\int_{E_{i}^{\xi}}\left|U \circ T g_{i}^{\xi}\right| d \lambda \geqslant m^{2}
$$

We choose $w_{1}^{\xi}, \ldots, w_{n_{\xi}}^{\xi}$ in $L^{\infty}(\lambda)$ such that

(i) $\operatorname{supp} w_{i}^{\xi} \subset E_{i}^{\xi}$,

(ii) $\left\|w_{i}^{\xi}\right\| \leqslant 1$ and

(iii) $\int_{w_{i}^{k}} L\left(g_{i}^{\xi}\right) d \lambda>m^{2} / 2$.

Since $\left\|P_{M}^{*} \circ L^{*}\right\| \leqslant 1$, we have that, for $\lambda_{1}, \lambda_{2}, \ldots, \lambda_{n_{\xi}}$ scalars,

$$
\left\|P_{M}^{*} \circ L^{*}\left(\sum_{i=1}^{n_{\xi}} \lambda_{i} w_{i}^{\xi}\right)\right\| \leqslant \sup _{1<i<n_{\xi}}\left|\lambda_{i}\right|
$$

Hence the set

$$
\Gamma_{i}^{\xi}=\left\{j: 1 \leqslant j \leqslant n_{\xi} \text { and }\left|P_{M_{\xi}}^{*} \circ L^{*}\left(w_{i}^{\xi}\right)\left(e_{j}^{\xi}\right)\right| \geqslant \varepsilon / 2(\kappa+1)\right\}
$$

has cardinality less than $[2(\kappa+1) / \varepsilon]+1$ and by Lemma 6.6 there is $A_{\xi} \subset$ $\left\{1, \ldots, n_{\xi}\right\}$ such that $\left|A_{\xi}\right|=2(\kappa+1)$ and $A_{\xi} \cap \Gamma_{i}^{\xi} \subset\{i\}$ for all $i \in A_{\xi}$.

With no loss of generality we assume that $A_{\xi}=\{1, \ldots, 2(\kappa+1)\}$. We set

$$
\left(x^{* *}\right)_{i}^{\xi}=\frac{1}{2}\left(U^{*} w_{i}^{\xi}-U^{*} w_{\kappa+1+i}^{*}\right)
$$

for all $1 \leqslant i \leqslant \kappa+1$ and from local reflexivity theorem we choose $\left\{x_{i}^{\xi}: 1 \leqslant i \leqslant \kappa\right\}$ such that

(i) $\left\|\sum_{i=1}^{\kappa} \lambda_{i} x_{i}^{\xi}\right\| \leqslant(1+\varepsilon) \sup \left|\lambda_{i}\right|$;

(ii) $\left\|x_{i}^{\xi}\right\| \leqslant 1$; and

(iii) $P_{M_{\xi}}^{*} \circ T^{*}\left(x_{i}^{\xi}\right)\left(e_{i}^{\xi}\right)>m^{2} / 8=\theta, \quad P_{M_{\xi}}^{*} \circ T^{*}\left(x_{i}^{\xi}\right)\left(e_{\kappa+i}^{\xi}\right)<-m^{2} / 8=\theta$, and $-\varepsilon /(\kappa+1)<P_{M_{\xi}}^{*} \circ T^{*}\left(x_{i}^{\xi}\right)\left(e_{j}^{\xi}\right)<\varepsilon /(\kappa+1)$ for all $1<j \leqslant 2(\kappa+1), j \neq i, j \neq \kappa$ $+i$.

Now setting $H_{\xi}=\left\{x_{1}^{\xi}, \ldots, x_{\kappa+1}^{\xi}\right\}$ and $h_{\xi}=M_{\xi}$ it follows that the pair $\left(\left\{H_{\xi}\right\}\right.$, $\left.\left\{h_{\xi}\right\}\right)$ is an elementary $\left(^{*}\right)$-pair, and the proof is complete. 
The following lemma gives a criterion for the existence of two (*)-pairs (elementary or not) $\left(F_{1}, f_{1}\right),\left(F_{2}, f_{2}\right)$ such that $\left(F_{1} \cup F_{2}, f_{1} \cup f_{2}\right)$ is also a (*)-pair.

6.7. Lemma. Let $\left\{\left(F_{n}, f_{n}\right): n<\omega\right\},(F, f)$ be $\left(^{*}\right)$-pairs such that

(i) $f_{n_{1}} \cap f_{n_{2}} \subset f$,

(ii) $\left|\cup f_{n}\right| \leqslant M,|\cup f| \leqslant M$ for some natural number $M$,

(iii) $P_{f \cup f_{n}}^{*} \circ T^{*} x=P_{f_{n}}^{*} \circ T^{*} x$ for all $x \in \cup F_{n}, n<\omega$.

Then there is an $n_{0}<\omega$ such that for every $n>n_{0}\left(F_{n} \cup F, f_{n} \cup f\right)$ is a (*)-pair.

PROof. Since for every $x \in \cup F$ the function $P_{f}^{*} \circ T^{*} x$ has finite range there is a positive number $\eta=\eta(F, f)$ such that, for every $H \in F, x_{1}, x_{2} \in H, x_{1} \neq x_{2}$ the following hold:

(i) $P_{f}^{*} \circ T_{x_{1}}\left[A_{x_{1}}\right] \subseteq[-1,-\theta-\eta)$,

(ii) $P_{f}^{*} \circ T_{x_{1}}\left[B_{x_{1}}\right] \subseteq(\theta+\eta, 1]$,

(iii) $P_{f}^{*} \circ T_{x_{2}}\left[A_{x_{1}} \cup B_{x_{1}}\right] \subseteq(n-\varepsilon / \kappa,-n+\varepsilon / \kappa)$, where $A_{x_{1}}, B_{x_{1}}$ are the associated sets of the element $x_{1}$, and $\kappa=|H|$.

From this and the assumption (iii) it follows that $\left(F_{n} \cup F, f_{n} \cup f\right)$ is a (*)-pair provided

$$
\left\|P_{f_{n} \cup f}^{*} \circ T^{*} x-P_{f}^{*} \circ T^{*} x\right\| \leqslant \eta \quad \text { when } x \in \cup F \text {. }
$$

Since $T^{*} x$ is an essential bounded function it follows that $T^{*} x$ is $L^{2}$-integrable and so there is an expression of it in $L^{2}$-norm as

$$
T^{*} x=\sum\left\{\alpha_{M} \Pi_{M}: M \in \mathscr{P}_{\omega}\left(\omega^{+}\right)\right\}
$$

Also the expression of $P_{\Lambda}^{*} \circ T^{*} x$, for some $\Lambda \subset \omega^{+}$, in \|\|$_{2}$ is

$$
P_{\Lambda}^{*} \circ T^{*} x=\sum\left\{\alpha_{M} \Pi_{M}: M \in \mathscr{P}_{\omega}(\Lambda)\right\} .
$$

From this and the assumption (i) it follows that for every $x \in \cup F$ the vectors $\left\{p_{f_{n} \cup f}^{*} \circ T^{*} x-P_{f}^{*} \circ T^{*} x: n<\omega\right\}$ are orthogonal. This implies that there is an $n_{0}$ such that for every $x \in \cup F$ and $n>n_{0}$

$$
\left\|P_{f_{n} \cup f}^{*} \circ T^{*} x-P_{f}^{*} \circ T^{*} x\right\|_{2}<\eta / 2^{M} .
$$

And since $\left|(\cup f) \cup\left(\cup f_{n}\right)\right| \leqslant 2 M$ it follows that

$$
\left\|P_{f \cup f_{n}}^{*} x-P_{f}^{*} x\right\|_{\infty} \leqslant 2^{\left|(\cup f) \cup\left(\cup f_{n}\right)\right| / 2}\left\|P_{f \cup f_{n}}^{*} x-P_{f}^{*} x\right\|_{2} \leqslant \eta
$$

for every $x \in \cup F$ and $n>n_{0}$. The proof is complete.

6.8. LEMMA. There is $\xi_{0}<\omega^{+}$such that if $n$ is a natural number and $(F, f) a$ ( $\left.^{*}\right)$-pair with $f \in \mathscr{P}_{\omega}\left(\omega^{+} \backslash \xi_{0}\right)$ then for each $\zeta<\omega^{+}$there are $h \in \mathscr{P}_{\omega}\left(\omega^{+} \backslash \zeta\right)$ and $H=\left\{x_{1}, \ldots, x_{n}\right\}$ such that

(i) if $\lambda_{1}, \ldots, \lambda_{n}$ are real numbers

$$
\left\|\sum_{j=1}^{n} \lambda_{j} x_{j}\right\| \leqslant(1+\varepsilon) \sup \left|\lambda_{j}\right| ;
$$

(ii) the pair ( $F \cup\{H\}, f \cup\{h\})$ is a $\left(^{*}\right)$-pair.

Proof. Assume the contrary; then there are

(i) a family $\left\{\left(F_{\xi}, f_{\xi}\right): \xi<\omega^{+}\right\}$of $\left(^{*}\right)$-pairs, 
(ii) a family $\left\{\zeta_{\xi}: \xi<\omega^{+}\right\}$of ordinals,

(iii) a family $\left\{n_{\xi}: \xi<\omega^{+}\right\}$of natural numbers such that $f_{\xi_{1}} \cap f_{\xi_{2}}=\varnothing$ for $\xi_{1}<\xi_{2}<\omega^{+}$and for each $\xi<\omega^{+}$there is no elementary $\left(^{*}\right)$-pair $\left(\left\{\boldsymbol{H}_{\xi}\right\},\left\{h_{\xi}\right\}\right)$ such that $\left|H_{\xi}\right|=n_{\xi}, h_{\xi} \in \mathscr{P}_{\omega}\left(\omega^{+} \backslash \zeta_{\xi}\right)$ and $\left(F_{\xi} \cup\left\{H_{\xi}\right\}, f_{\xi} \cup\left\{h_{\xi}\right\}\right)$ is a $\left(^{*}\right)$-pair.

With no loss of generality, by passing if it is necessary to a subfamily with the same cardinality, we assume that for all $\xi<\omega^{+},\left|\cup f_{\xi}\right|=\kappa$ and $n_{\xi}=n$.

Let $\left\{\xi_{1}<\xi_{2}<\cdots<\xi_{n}<\ldots: n<\omega\right\}$ be a sequence of ordinals. For each $\xi_{n}$ we set $N_{n}$ to be the countable subset of $\omega^{+}$on which depends the function $\left\{T^{*} x\right.$ : $\left.x \in \cup F_{\xi_{n}}\right\}$ and we choose an ordinal $\zeta_{0}$ such that $\cup_{n=1}^{\omega} N_{\xi} \subset \zeta_{0}$, $\sup _{n<\omega} \zeta_{\xi_{n}} \leqslant \zeta_{0}$ and $\cup_{n=1}^{\omega}\left(\cup f_{\xi_{n}}\right) \subset \zeta_{0}$. Then by Lemma 6.5 there is an elementary $\left(^{*}\right)$-pair ( $\{H\},\{h\}$ ) such that $h \subset \omega^{+} \backslash \zeta_{0},|H|=n$ and $H$ satisfies (i). Also since $N_{\xi_{n}} \cap h$ $=\varnothing$ from Lemma it follows that for every $n<\omega$ and $x \in \cup F_{\xi}$

$$
P_{f_{\xi_{n}} \cup\{h\}}^{*} \circ T^{*} x=P_{f_{\xi_{n}}}^{*} \circ T^{*} x .
$$

So from Lemma 6.6 it follows that there is a natural number $n_{0}$ such that $\left(F_{\xi_{0}} \cup\{H\}, f_{\xi_{0}} \cup\{h\}\right)$ is a $\left(^{*}\right)$-pair, a contradiction, and the proof is complete.

6.9. Construction of $(P,<)$. Using transfinite induction we choose

(1) a family $\left\{P_{\xi}: \xi<\omega^{+}\right\}$and

(2) for every $\xi<\omega^{+}$a family $\left\{\mathcal{\vartheta}_{\xi}^{o}: \xi \leqslant \sigma<\omega^{+}\right\}$such that:

(1.a) For every $\xi<\omega^{+}, P_{\xi}$ is a set of pairs $(F, f)$ each of which is a $\left(^{*}\right)$-pair.

(1.b) If $(F, f),(G, g)$ belong to $P_{\xi}$ and $(F \cup G, f \cup g)$ is a $\left(^{*}\right)$-pair, then $\left(F \cup G, f \cup g\right.$ ) also belongs to $P_{\xi}$.

(1.c) Each $P_{\xi}$ is a countable set.

(1.d) For $\xi_{1}<\xi_{2}, P_{\xi_{1}} \subset P_{\xi_{2}}$.

(1.e) For every $\xi<\omega^{+}, \zeta \leqslant \sigma<\xi$, ข $\mathcal{\zeta}_{\zeta}^{\sigma} \subset P_{\xi}$.

(1.f) If $\xi<\omega^{+},(F, f) \in P_{\xi}$ and $H \in F$ where $H=\left\{x_{1}, \ldots, x_{n}\right\}$, then for all $\lambda_{1}, \lambda_{2}, \ldots, \lambda_{n}$ scalars we have

$$
\left\|\sum_{j} \lambda_{j} x_{j}\right\| \leqslant(1+\varepsilon) \sup _{1<j<n}\left|\lambda_{j}\right| .
$$

(2.a) If $\xi \leqslant \zeta<\omega^{+}$and $\xi$ has the form $\sigma+\kappa$ for some limit ordinal $\sigma$ and some finite ordinal $\kappa$, then for each element $(F, f)$ of $Q_{\xi}^{\xi}$ there is $H \in F$ such that $|H| \geqslant \kappa+1$.

(2.b) For every $\xi<\omega^{+}$the set $\mathcal{Q}_{\xi}^{\xi}$ has the property: for each $(F, f) \in P_{\xi}$ there is $(G, g) \in \mathcal{Q}_{\xi}^{\xi}$ such that

$$
G=F \cup\{H\}, \quad g=f \cup\{h\} .
$$

(2.c) For every $\xi<\zeta<\omega^{+}$and $(F, f) \in \mathcal{Q}_{\xi}^{\zeta}$ there is $(G, g) \in \mathcal{Q}_{\xi}^{\zeta}$ such that $G=F \cup\{H\}, g=f \cup\{h\}$.

(2.d) If $\xi_{1}<\xi_{2}$ and $\xi_{1} \leqslant j_{1}<\omega^{+}, \xi_{2} \leqslant j_{2}<\omega^{+}$then $\mathcal{Q}_{\xi_{1}}^{\xi_{1}} \cap \mathcal{Q}_{\xi_{2}}^{\xi_{2}}=\varnothing$. (The inductive choice uses Lemma 6.8 and is straightforward.)

We set $P=\cup_{\xi<\omega^{+}} P_{\xi}$ and for each $\xi<\omega^{+} \mathcal{Q}_{\xi}=\cup_{\sigma=\xi}^{\omega^{+}} \mathcal{U}_{\xi}^{\sigma}$, and for $(F, f)$, $(G, g)$ in $P$ we set $(G, g)<(F, f)$ iff $F \subset G$ and $f \subset g$. Obviously the set $(P,<)$ is a partially ordered set and each $\mathcal{Q}_{\xi}$ is a dense subset of $P$.

6.10. Lemma. The set $(P,<)$ has the c.c.c. 
Proof. We choose a family $\left\{\left(F_{\xi}, f_{\xi}\right): \xi<\omega^{+}\right\}$of pairwise distinct elements of $P$. From the Erdös-Rado $\Delta$-system lemma (Lemma 1.1) we can assume that there are $F, f$ such that for every $\xi_{1}<\xi_{2}$

$$
F_{\xi_{1}} \cap F_{\xi_{2}}=F \text { and } f_{\xi_{1}} \cap f_{\xi_{2}}=f .
$$

Let us assume that $F \neq \varnothing, f \neq \varnothing$ (if $F=\varnothing, f=\varnothing$ the proof is analogous). We let $N$ be the subset of $\omega^{+}$on which the set $\left\{T^{*} x: x \in H\right.$ and $\left.H \in F\right\}$ depends, and for each $\xi<\omega^{+}$we denote by $N_{\xi}$ the subset of $\omega^{+}$on which the set $\left\{T^{*} x: x \in H\right.$ and $\left.H \in F_{\xi} \backslash F\right\}$ depends.

We choose $A \subset \omega^{+}$such that

(i) $|A|=\omega^{+}$,

(ii) there is $M$ such that $\left|\cup\left\{h: h \in f_{\xi}\right\}\right| \leqslant M$ holds for all $\xi \in A$,

(iii) setting $f_{\xi}^{\prime}=f_{\xi} \backslash f$ then $N \cap\left(\cup f_{\xi}^{\prime}\right)=\varnothing$ and,

(iv) if $\xi_{1}<\xi_{2}$ elements of $A, N_{\xi_{1}} \cap\left(\cup f_{\xi_{2}}^{\prime}\right)=\varnothing$.

We choose $\xi_{0} \in A$ such that the set $\Lambda=\left\{\zeta \in A: \zeta<\xi_{0}\right\}$ is infinite. By Lemma 2.4 it follows that for every $\zeta \in \Lambda$ and $x \in \cup F_{\zeta}$

$$
P_{f_{5} \cup f_{f_{0}}}^{*} \circ T^{*} x=P_{f_{5}}^{*} \circ T^{*} x
$$

and from Lemma 6.5 it follows that for all except finitely many $\zeta$ elements of $\Lambda$ $\left(F_{\zeta} \cup F_{\xi_{0}}, f_{\zeta} \cup f_{\xi_{0}}\right)$ is a $\left(^{*}\right)$-pair.

This completes the proof of the lemma.

Proof of Theorem 6.3. Using Martin's axiom we choose a filter $\mathscr{F}$ subset of $P$ such that $\mathscr{F} \cap \mathscr{Q}_{\xi} \neq \varnothing$ for all $\xi<\omega^{+}$. We prove that the closed linear space which is generated from the family $\{x \in X: \exists(F, f) \in \mathscr{F}$ and $H \in F$ with $x \in H\}$ is isomorphic to $Z_{\omega^{+}}$.

We note first, by the inductive assumption (2.a), that for each $n<\omega$ there are uncountably many $H$ such that $|H| \geqslant n, H \in F$, and $(F, f) \in \mathscr{F}$. Also, if $\left\{\left(F_{i}, f_{i}\right)\right.$ : $i=1,2, \ldots, n\}$ is a finite subset of $H_{1}, H_{2}, \ldots, H_{n}$ and is such that $H_{i} \in F_{i}$, $H_{i_{1}} \neq H_{i_{2}}$ for $1 \leqslant i_{1}<i_{2} \leqslant n$, and $y_{1}, y_{2}, \ldots, y_{n}$ are nonzero elements of $X$, such that $y_{i}=\sum_{j=1}^{\kappa_{i}} \lambda_{i}^{j} x_{i}^{j}$ where $x_{i}^{j_{1}} \neq x_{i}^{j_{2}}$ for $j_{1} \neq j_{2}$ and $x_{i}^{j} \in H_{i}$ for all $j=1, \ldots, \kappa_{i}$, then we have

$$
(1+\varepsilon) \sum_{i=1}^{n} \max _{1<j<\kappa_{i}}\left|\lambda_{i}^{j}\right| \geqslant\left\|\sum_{i=1}^{n} y_{i}\right\| \geqslant \frac{\theta}{2} \sum_{i=1}^{n} \max _{1<j<\kappa_{i}}\left|\lambda_{i}^{j}\right| .
$$

In fact, the first part of the inequality is a direct consequence of the inductive assumption (1.f).

For the second part, since $\mathscr{F}$ is a filter there is an $(F, f) \in \mathscr{F}$ such that $H_{i} \in F$ for all $i=1,2, \ldots, n$ and for each $1 \leqslant i \leqslant n$ we choose $j_{i}$ such that $\left|\lambda_{i}^{j_{i}}\right|=$ $\sup _{1 \leqslant j \leqslant \kappa_{i}}\left|\lambda_{i}^{j}\right|$. Then, setting

$$
K=\bigcap_{i=1}^{n} \varepsilon_{i} A_{x_{i} / i}^{f}
$$

where

$$
\begin{aligned}
\varepsilon_{i} A_{x_{i}^{j_{i}}}^{f_{i}} & =A_{x_{i}^{j_{i}}}^{f} \quad \text { if }\left|\lambda_{i}^{j_{i}}\right|=-\lambda_{i}^{j_{i}}, \\
& =B_{x_{i} j_{i}}^{f} \quad \text { otherwise, }
\end{aligned}
$$


it follows that

$$
\begin{aligned}
\left\|\sum_{i=1}^{n} y_{i}\right\| & \geqslant\left\|\sum_{i=1}^{n} P_{f}^{*} \circ T^{*}\left(y_{i}\right)\right\| \geqslant \frac{1}{\mu(\mathrm{K})}\left|\sum_{i=1}^{n} \int_{\mathbf{K}} P_{f}^{*} \circ T^{*}\left(y_{i}\right) d \mu\right| \\
& \geqslant(\theta-\varepsilon) \sum_{i=1}^{n}\left|\lambda_{i}^{j_{x}}\right| \geqslant \frac{\theta}{2} \sum_{i=1}^{n} \sup _{1<j<\kappa_{i}}\left|\lambda_{i}^{j}\right| .
\end{aligned}
$$

The proof of the theorem is now complete.

6.11. THEOREM. Assume Martin's axiom and the negation of the continuum hypothesis. Let $X$ be a Banach space such that there is a norm one, one-to-one operator $T: L^{1}\{-1,1\}^{\omega^{+}} \rightarrow X^{*}$. Then $l_{\omega^{+}}^{1}$ is isomorphic to a subspace of $X$.

An outline of the proof of this theorem is as follows.

6.12. Definition. Let $F$ be a finite subset of $X$ and $f$ a finite subset of $\omega^{+}$. The pair $(F, f)$ is a $\left(^{*}\right)$-pair iff $|F|=|f|$ and there is an enumeration of $f$, say

$$
f=\left\{\xi_{x}: x \in F\right\} \text {, }
$$

such that if

$$
A_{x}^{f}=P_{f}^{*} \circ T^{*}(x)^{-1}\left[-\|x\|,-\frac{1}{2}\right), \quad B_{x}^{f}=P_{f}^{*} \circ T^{*}(x)^{-1}\left[\frac{1}{2},\|x\|\right),
$$

then

$$
A_{x}^{f}=\{-1\} \times\{-1,1\}^{f \backslash\left\{\xi_{x}\right\}}, \quad B_{x}^{f}=\{1\} \times\{-1,1\}^{f \backslash\left\{\xi_{x}\right\}}
$$

6.13. LemMA. Let $(F, f)$ be a $\left(^{*}\right)$-pair. Then there are $x_{(F, f)}$ and $\xi_{(F, f)}<\omega^{+}$such that $\left(F \cup\left\{x_{(F, f)}\right\}, f \cup\left\{\xi_{(F, f)}\right\}\right)$ is also a $\left(^{*}\right)$-pair.

Proof. For every $x \in F$ we set $N_{x}$ to be the countable subset of $\omega^{+}$on which depends the function $T^{*} x$ and $N_{F}=\cup_{x \in F} N_{x}$. We choose $\xi_{(F, f)}<\omega^{+}$such that $\xi_{(F, f)} \notin N_{F}$. Since $T^{*} X$ is $w^{*}$ dense into $L^{\infty}\{-1,1\}^{\omega^{+}}$it follows that the linear operator $P_{f^{\prime}}^{*} \circ T^{*}: X \rightarrow L^{\infty}\{-1,1\}^{f^{\prime}}$ is onto, where $f^{\prime}=f \cup\left\{\xi_{(F, f)}\right\}$. So there is $x_{(F, f)} \in X$ such that $P_{f}^{*} \circ T^{*}\left(x_{(F, f)}\right)=\Pi_{\left\{\xi_{(F, f)}\right.}$. And since $P_{f}^{*} \circ T^{*} x=P_{f^{\prime}}^{*} \circ T^{*} x$ for all $x \in F$, by Lemma 2.4, we have that the pair $\left(x_{(F, f)}, \xi_{(F, f)}\right)$ is the desired one.

Proof OF 6.11. Using Lemma 6.13 we construct a partially ordered set $(P,<)$ in the same way as in 6.9 and using similar arguments as in Lemma 6.10 we prove that $P$ is c.c.c. From Martin's axiom it follows that there is a filter $\mathcal{F}$ with uncountably many elements. For every $n<\omega$ we set

$$
E_{n}=\{x \in X: \exists(F, f) \in \mathscr{F} \text { with } x \in F \text { and }\|x\| \leqslant n\} .
$$

Since $\mathcal{F}$ is uncountable there is $n_{0}<\omega$ such that $\left|E_{n}\right|=\omega^{+}$.

It is easy to see that the elements of $E_{n}$ form a basis of $l_{\omega^{+}}^{1}$.

7. In this last section of the paper we give some applications of the results of the previous sections. So under Martin's axiom and the negation of the continuum hypothesis we prove a conjecture of Rosenthal given in [14] about the isomorphic structure of conjugate $L^{1}(\lambda)$ spaces. Also, we extend another result of Rosenthal (Theorem 5.1 of [14]) about the structure of the conjugate of the spaces $C(S)$. 
7.1. Theorem. Assume M.A. and $\rceil$ C.H. Let $\lambda$ be a measure such that $L^{1}(\lambda)$ is isomorphic to $X^{*}$ for some infinite dimensional Banach space $X$. Then there is a set $\Gamma$ and a function $\phi: \Gamma \rightarrow$ Card such that $\phi(\gamma)=0$ or $\phi(\gamma)$ is an infinite cardinal and such that

$$
L^{1}(\lambda) \cong\left(\sum_{\gamma \in \Gamma} \oplus L^{1} \phi(\gamma)\right)_{1}
$$

where

$$
\begin{gathered}
L^{1} \phi(\gamma) \cong \mathbf{R} \quad \text { if } \phi(\gamma)=0 \text { and } \\
L^{1} \phi(\gamma) \cong\left(\sum_{2^{\alpha}} \oplus L^{1}\{-1,1\}^{\alpha}\right)_{1} \quad \text { if } 0 \neq \phi(\gamma)=\alpha
\end{gathered}
$$

We prove first the following auxiliary results.

7.2. Lemma. Let $\Delta$ be a set and $\phi: \Delta \rightarrow$ Card be a mapping such that, for all $\alpha \in \phi(\Delta),|\{\delta \in \Delta: \phi(\delta) \geqslant \alpha\}| \geqslant 2^{\alpha}$. Then there is a disjoint family $\left(\Delta_{\alpha}\right)_{\alpha \in \phi(\Delta)}$ of subsets of $\Delta$ such that for all $\alpha$ we have

$$
\left|\Delta_{\alpha}\right| \geqslant \max \left\{2^{\alpha},\left|\phi^{-1}(\alpha)\right|\right\}
$$

and, for all $\delta \in \Delta_{\alpha}, \phi(\delta) \geqslant \alpha$.

Proof. There are two cases:

Case 1. For every $\alpha \in \phi(\Delta)$ there is $\beta(\alpha) \in \phi(\Delta)$ with $\beta(\alpha) \geqslant \alpha$ and $\left|\phi^{-1}(\beta(\alpha))\right|$ $\geqslant 2^{\alpha}$.

In this case we define a function $f: \phi(\Delta) \rightarrow \phi(\Delta)$ with the rule

$$
f(\alpha)=\min \left\{\beta: \beta \geqslant \alpha \text { and }\left|\phi^{-1}(\beta)\right| \geqslant 2^{\alpha}\right\} .
$$

For every $\beta \in \phi(\Delta)$ with $f^{-1}(\beta) \neq \varnothing$ we define a decomposition of $\phi^{-1}(\beta)$ into sets $\left\{\Delta_{\alpha}: \alpha \in f^{-1}(\beta)\right\}$ such that $\left|\Delta_{\alpha}\right|=\max \left\{2^{\alpha},\left|\phi^{-1}(\alpha)\right|\right\}$. It is easy to see that the family $\left\{\Delta_{\alpha}: \alpha \in f^{-1}(\beta), \beta \in f(\phi(\Delta))\right\}$ is as desired.

Case 2. There is an $\alpha \in \phi(\Delta)$ such that $2^{\alpha}>\left|\phi^{-1}(\beta)\right|$ for all $\beta>\alpha$ and $\beta \in \phi(\Delta)$.

We define a decomposition of $\phi(\Delta)$ into $\Delta_{1}, \Delta_{2}$ sets such that $\Delta_{1}=\left\{\alpha\right.$ : $\left|\phi^{-1}(\alpha)\right|$ $\left.>2^{\alpha}\right\}$ and $\Delta_{2}=\Delta \backslash \Delta_{1}$.

From our assumptions it follows that $\left|\Delta_{2}\right| \geqslant 2^{\alpha}$ for all $\alpha \in \Delta_{2}$. So there is a pairwise disjoint family of subsets of $\Delta_{2}$, say $\left\{E_{\alpha}: \alpha \in \Delta_{2}\right\}$, with the properties $\left|E_{\alpha}\right| \geqslant 2^{\alpha}$ and, for every $\beta \in E_{\alpha}, \beta \geqslant \alpha$.

Now the family $\left\{\phi^{-1}(\alpha): \alpha \in \Delta_{1}\right\} \cup\left\{\phi^{-1}\left(E_{\alpha}\right): \alpha \in \Delta_{2}\right\}$ is as desired and the proof is complete.

7.3. Lemma. Let $\alpha$ be an infinite cardinal and $\left\{\alpha_{\gamma}: \gamma \in \Gamma\right\}$ a family of cardinals. Assume, also, that there is

$$
T:\left(\sum_{\xi<2^{\alpha}} \oplus L_{\xi}^{1}\{-1,1\}^{\alpha}\right)_{1} \rightarrow\left(\sum_{\gamma \in \Gamma} \oplus L^{1}\{-1,1\}^{\alpha_{\gamma}}\right)_{1},
$$

an isomorphic embedding. Then the set $\Delta=\left\{\gamma \in \Gamma: \alpha_{\gamma} \geqslant \alpha\right\}$ has cardinality greater than or equal to $2^{\alpha}$. 
Proof. We first recall a result of Lindenstrauss ${ }^{2}$ that states that for every $\alpha$ infinite cardinal the space $L^{1}\{-1,1\}^{\alpha}$ is isomorphic to a subspace of a space $\left(\Sigma_{\gamma \in \Gamma} \oplus L^{1}\{-1,1\}^{\alpha_{\gamma}}\right)_{1}$ provided that there is $\gamma \in \Gamma$ such that $\alpha_{\gamma} \geqslant \alpha$.

This result implies that for every $\xi<2^{\alpha}$ there is $x_{\xi} \in L_{\xi}^{1}\{-1,1\}^{\alpha}$ such that $\left\|x_{\xi}\right\|=1,\left\|P_{\Gamma \backslash \Delta} \circ T\left(x_{\xi}\right)\right\|<4\left\|T^{-1}\right\|^{-1}$, where

$$
P_{\Gamma \backslash \Delta}:\left(\sum_{\gamma \in \Gamma} \oplus L^{1}\{-1,1\}^{\alpha_{\gamma}}\right)_{1} \rightarrow\left(\sum_{\gamma \in \Gamma \backslash \Delta} \oplus L^{1}\{-1,1\}^{\alpha_{\gamma}}\right)_{1}
$$

is the usual projection.

There is, also, $E_{\xi} \subset \Delta$ finite such that

$$
\left\|P_{\Delta \backslash E_{\xi}} \circ T\left(x_{\xi}\right)\right\|<4\left\|T^{-1}\right\|^{-1} .
$$

Assuming now that $|\Delta|<2^{\alpha}$ we find $E \subset \Delta$ finite and $G \subset 2^{\alpha}$ with $|G|=\omega^{+}$ such that, for every $\xi \in G, E_{\xi}=E$.

We claim that the family $\left\{P_{E} \circ T\left(x_{\xi}\right): \xi \in G\right\}$ is equivalent to the usual basis of $l_{\omega^{+}}^{1}$ although it is contained into the weakly compactly generated space $\left(\Sigma_{\gamma \in E} \oplus L^{1}\{-1,1\}^{\alpha_{\gamma}}\right)_{1}$, a contradiction.

Indeed, for $\xi_{1} \neq \cdots \neq \xi_{n}$ elements of $G$ and $r_{1}, \ldots, r_{n}$ real numbers, we have

$$
\begin{aligned}
\left\|P_{E} \circ T\left(\sum_{i=1}^{n} r_{1} x_{\xi}\right)\right\| & \geqslant\left\|T\left(\sum_{i=1}^{n} r_{i} x_{\xi}\right)\right\|-\left\|P_{\Gamma \backslash E} \circ T\left(\sum_{i=1}^{n} r_{i} x_{\xi}\right)\right\| \\
& \geqslant \frac{1}{\left\|T^{-1}\right\|} \sum_{i=1}^{n}\left|r_{i}\right|-\frac{1}{2\left\|T^{-1}\right\|} \sum_{i=1}^{n}\left|r_{i}\right| \\
& =\frac{1}{2\left\|T^{-1}\right\|} \sum_{i=1}^{n}\left|r_{i}\right| .
\end{aligned}
$$

The proof of the lemma is now complete.

Proof OF TheOREM 7.1. Let $\left\{\mu_{\delta}: \delta \in \Delta\right\}$ be a family of measures such that

$$
L^{1}(\lambda)=\left(\sum_{\delta \in \Delta} \oplus L^{1}\left(\mu_{\delta}\right)\right)_{1}
$$

and for each $\delta \in \Delta, L^{1}\left(\mu_{\delta}\right)=\mathbf{R}$ or $L^{1}\left(\mu_{\delta}\right)=L^{1}\{-1,1\}^{\alpha_{\delta}}$ for some $\alpha_{\delta}>\omega$.

We set

$$
\Delta_{0}=\left\{\delta \in \Delta: L^{1}\left(\mu_{\delta}\right)=\mathbf{R}\right\}, \quad \Delta_{1}=\Delta \backslash \Delta_{0} .
$$

Let $\phi: \Delta_{1} \rightarrow$ Card be a function defined by the rule $\phi(\delta)=\alpha_{\delta}$. From Lemma 7.3 and Theorems 3.1 and 6.11 it follows that $\phi$ satisfies the assumptions of Lemma 7.2; so there is a family of pairwise disjoint subsets of $\Delta_{1}$, let $\left\{\Delta_{\alpha}: \alpha \in \phi\left(\Delta_{1}\right)\right\}$, with $\left|\Delta_{\alpha}\right|=\max \left\{2^{\alpha},\left|\phi^{-1}(\alpha)\right|\right\}$. Setting

$$
L^{1}\left(\lambda_{1}\right)=\left(l^{1}\left(\Delta_{0}\right) \oplus \sum_{\alpha \in \phi\left(\Delta_{1}\right)} \oplus\left(\sum_{\left|\phi^{-1} \alpha\right|} \oplus\left(C\{-1,1\}^{\alpha}\right)^{*}\right)\right)_{1}
$$

${ }^{2}$ Cf. E. Lacey, The isometric theory of classical Banach spaces, Springer-Verlag, Berlin and New York, Theorem 14, p. 130. 
it is easy to check that

$$
L^{1}\left(\lambda_{1}\right) \underset{\perp}{\hookrightarrow} L^{1}(\lambda) \underset{\perp}{\hookrightarrow} L^{1}\left(\lambda_{1}\right)
$$

and from Pekzynski's decomposition lemma, it follows that $L^{1}\left(\lambda_{1}\right) \cong L^{1}(\lambda)$. The proof is complete.

7.4. REMARK. As the referee remarked, Theorem 7.1 itself holds with no set-theoretical assumption, provided one assumes that $L^{1}\{-1,1\}^{\omega^{++}}$embeds into $L^{1}(\lambda)$. Indeed, in this case, Theorem 3.1 is enough to establish that the set $\Delta_{1}$ which appears in the proof of Theorem 7.1 satisfies the condition of Lemma 7.2.

7.5. Theorem. Assume M.A. and $\rceil$ C.H. Let $X$ be a complemented subspace of $a$ space $C(S)$ with $\operatorname{dim} X=\alpha$; then the following are equivalent:

(a) $l_{\alpha}^{1}$ is isomorphic to a subspace of $X$,

(b) $X^{*}$ is isomorphic to the space $\left(\Sigma_{2^{\alpha}} \oplus L^{1}\{-1,1\}^{\alpha}\right)_{1}$.

If furthermore $X$ is an injective Banach space, then conditions (a), (b) are equivalent without any additional set-theoretical assumption.

Proof. From [13] and [18] it follows that (a) is equivalent to (b) in the case $\alpha=\omega$.

Let us assume that $\alpha>\omega$.

(b) $\Rightarrow$ (a). It follows immediately from Theorem 3.1 if $\alpha>\omega^{+}$, and from Theorem 6.11 if $\alpha=\omega^{+}$.

(a) $\Rightarrow$ (b). Let $\Lambda=\left\{x_{\xi}: \xi<\alpha\right\}$ be a dense subset of $X$ and we let $\mathcal{T}_{\Lambda}$ be the smallest topology on $S$ for which the elements of $\Lambda$ are continuous functions and let $\Omega$ be the $T_{2}$ quotient space of $\left(S, T_{\Lambda}\right)$ which is defined by the relation $s_{1} \sim s_{2}$ iff $x_{\xi}\left(s_{1}\right)=x_{\xi}\left(s_{2}\right)$ for all $\xi<\alpha$.

Then there is a natural continuous onto map $\phi: S \rightarrow \Omega$.

If $\phi^{0}: C(\Omega) \rightarrow C(S)$ is the natural isometric embedding which is defined from $\phi$, then it follows that $\phi^{0}(C(\Omega))$ contains the space $X$ and $\operatorname{dim} C(\Omega)=\alpha$. So we can assume that $X$ is a complemented subspace of a space $C(\Omega)$ with $\operatorname{dim} C(\Omega)=$ $\operatorname{dim} X=\alpha$. Since $l_{\alpha}^{1}$ is isomorphic to a subspace of $X$, from Theorem 5.1 of [14] it follows that

$$
X^{*} \hookrightarrow M(\Omega) \cong\left(\sum_{2^{\alpha}} \oplus L^{1}\{-1,1\}^{\alpha}\right)_{1}
$$

On the other hand from $l_{\alpha}^{1}$ embedding into $X$ we have that $L^{1}\{-1, \cdot 1\}^{\alpha}$ is isomorphic to a subspace of $X^{*}[13]$ and since $X^{*}$ is isomorphic to a subspace of $L^{1}(\lambda)$ for some measure $\lambda$, it follows that $Z_{\alpha}$ is isomorphic to a subspace of $X$, by Theorems 4.2 and 6.3. So from [7] we have that

$$
\left(\sum_{2^{\alpha}} \oplus L^{1}\{-1,1\}^{\alpha}\right)_{1} \stackrel{\perp}{\hookrightarrow} X^{*} .
$$

From (1) and (2) and Pełczynski's decomposition lemma we have the desired result.

For the last part of the statement of the theorem, about injective Banach spaces, we observe that in the case where $\operatorname{dim} X=\omega^{+}$the result follows from the fact that $l_{\omega}^{\infty}$ is isomorphic to a (complemented) subspace of $X$, while for the case $\alpha>\omega^{+}$we do not use any additional set-theoretical assumptions. 
7.6. Remark. From Theorem 7.3 it follows that questions (b) and (c) in Problem 7 of [14] are equivalent. Also, in [1] we prove that, under G.C.H., if $X$ is an injective Banach space, then $(\operatorname{dim} X)^{\omega}=\operatorname{dim} X$, which gives an affirmative answer to the question (a) of the same problem. Finally, without any set-theoretical assumption, in [2] we prove that question (b) implies question (a).

\section{REFERENCES}

1. S. Argyros, On dimension of injective Banach spaces, Proc. Amer. Math. Soc. 78 (1980), 267-268.

2. Weak compactness in $L^{1}(\lambda)$ and injective Banach spaces, Israel J. Math. 37 (1980), 21-33.

3. S. Argyros and S. Negrepontis, Universal embeddings of $l_{\alpha}^{1}$ into $C(X)$ and $L^{\infty}(\mu)$, Colloq. Math. Soc. Janós Bolyai Topology, vol. 23, Budapest, 1978.

4. W. W. Comfort and S. Negrepontis, The theory of ultrafilters, Band 211, Springer-Verlag, Berlin, 1974.

5. L. Dor, On projection $L^{1}$, Ann. of Math. (2) 102 (1978), 463-474.

6. J. Hagler, On the structure $S$ and $C(S)$ for $S$ dyadic, Trans. Amer. Math. Soc. 214 (1975), 415-427.

7. J. Hagler and C. Stegall, Banach spaces whose duals contain complemented subspaces isomorphic to $C[0,1]^{*}$, J. Funct. Anal. 13 (1973), 233-251.

8. A. Hajnal, Proof of a conjecture of S. Ruziewicz, Fund. Math. 50 (1961), 123-128.

9. R. Haydon, On Banach spaces which contain $l^{1}(\tau)$ and types of measures on compact spaces, Israel J. Math. 28 (1977), 313-324,

10. __ On dual $L^{1}$ spaces and injective bidual Banach spaces, Israel J. Math. 7 (1978), 142-152.

11. W. B. Johnson, H. P. Rosenthal and M. Zippin, On bases finite dimensional decompositions and weaker structures in Banach spaces, Israel J. Math. 9 (1971), 488-506.

12. A. PeYczynski, Projections in certain Banach spaces, Studia Math. 19 (1960), 209-228.

13. __ On Banach spaces containing $L^{1}(\mu)$, Studia Math. 30 (1968), 231-246.

14. H. P. Rosenthal, On injective Banach spaces and the spaces $L^{\infty}(\mu)$ for finite measures $\mu$, Acta Math. 127 (1970), 205-248.

15. , On relatively disjoint families of measures with some applications to Banach space theory, Studia Math. 37 (1970), 13-36.

16. M. E. Rudin, Martin's axiom, Handbook of Mathematical Logic, (J. Barwise, ed.), North-Holland, Amsterdam, 1977, pp. 481-502.

17. T. W. Starbird, Subspaces of $L^{1}$ containing $L^{1}$, Dissertation, Univ. of California, Berkeley, 1976.

18. C. Stegall, Banach spaces whose duals contain $l^{\prime}(\Gamma)$ with applications to the study of dual $L^{1}(\mu)$ spaces, Trans. Amer. Math. Soc. 17 (1973), 463-477.

Chair I of Mathematical Analysis, Athens University, Athens 621, Greece 